\title{
Risk aversion, prudence and temperance: it is a matter of gap between moments
}

\author{
Annarita Colasante ${ }^{1}$ and Luca Riccetti ${ }^{2}$ \\ ${ }^{1}$ University Jaume I, Economics Department, Castellon de la Plana (Spain) \\ ${ }^{2}$ University of Macerata, Economics and Law Department, Macerata (Italy)
}

\begin{abstract}
Higher order risk preferences are important determinants of choices under uncertainty. We build a questionnaire different from usually adopted ones: our questionnaire is simpler in order to reduce the number of random choices, and it includes questions with largely diversified stake sizes to observe different gaps between moments. Moreover, we collect results from a large and heterogeneous population to provide more general and unbiased results. Our results confirm the preference of the majority of the respondents for higher odd and lower even moments of the expected return distribution. However, we highlight three features: (i) the importance of the gap between the values of the corresponding moments of the two choices, (ii) the behavioral change in presence of a positive/zero/negative expected value, (iii) the huge heterogeneity in behaviors, also due to the complexity of the choice as an important driver of the propensity to switch from choosing on the basis of preferences to choo sing randomly. We also find that age and geographical location are important determinants of risk propensity.
\end{abstract}

Keywords: behavioural finance, experiment, choice under uncertainty, moments of return distribution, utility function.

JEL classification codes: C91, D01, D81, D90, G40.

Acknowledgments: We are grateful for helpful comments and useful suggestions to participants in the " $24^{\text {th }}$ International Conference on Computing in Economics and Finance" (CEF 2018), organized at University Cattolica del Sacro Cuore, Milan, June $19^{t h}-21^{\text {st }} 2018$. 


\section{Introduction}

An important economic literature strand studies the problem of economic choices under uncertainty. This is a very relevant topic because of its implications in understanding the behavior of subjects, for instance in the portfolio allocation process of investors. Economists and practitioners often model these choices assuming that: (i) subjects are rational and maximize their utility function (expected utility theory); (ii) the utility of subjects is a function of the expected mean and variance of the return distribution. This approach is based on the pioneer and well-known mean-variance approach developed by Harry Markowitz (Markowitz, 1952). This theory is based on the idea that investors face a dilemma between getting the highest possible earning and taking the minimum risk. The mean-variance approach formalizes this dilemma and provides a possible solution by computing the so-called efficient frontier. Even if this theory sometimes provides a $g$ ood approximation of individual financial decisions, subsequent analyses show that not only is portfolio decision a more complex problem, but also that investors are more complex than traditional theory assumed. On one hand, psychologists discover that investors display different biases in making their decisions: for example, they can over or under estimate probability (Kahneman and Tversky, 1972) and are strongly influenced by they way a problem is framed (Tversky and Kahneman, 1981); therefore, subjects can not be defined as perfectly rational. On the other hand, the utility function considers also higher (non standardized, central) moments of the return distribution. This last assumption could be "recovered" if the asset returns are Normally distributed and, as a consequence, it is sufficient to focus just on the first and second moment of the distribution (i.e., mean and variance). Samuelson [1970] showed that higher moments of the return distribution are negligible if the probability distribution of wealth is compact. Under this condition, the mean-variance model provides a good approximation of portfolio selection. However, Samuelson [1970] and a long list of empirical papers (see, for instance, Arditti, 1967; Kon, 1984; Peiro, 1999; Harvey and Siddique, 2000; Jondeau and Rockinger, 2003) provide evidence that asset return distributions are usually very different from a Normal one showing, for example, both skewness and excess kurtosis far from zero. Scott and Horvath [1980] and Kane [1982] underlined the importance of higher moments of the return distribution on investors' decisions, so higher order risk preferences are important determinants of subjects' behavior. Laboratory experiments, as well as surveys, turn out to be effective tools to directly elicit subjects' risky preferences. However, the interest of behavioral economists for higher order risk preferences is more recent, and in this field a specific terminology is used that is almost disconnected from the financial literature strand that analyses the influence of higher moments of the return distribution. Indeed, in the field of behavioral finance we refer to preference for third and fourth moments as prudence (Kimball, 1990) and temperance (?, ?) respectively (see Appendix B for an in-depth discussion on the terminology). In the literature there are quite few experimental works that investigate these aspects and almost all reach the same conclusion: the majority of people are prudent, while temperance is a weaker feature.

The main aim of this work is to investigate how subjects take into account the first four moments of the return distribution in making risky decisions. To reach this goal we built a questionnaire with a total of 14 risky lotteries, reported in Appendix A. Moreover, we performed a large set of analyses. First, to address a subset of research questions related to such decisions, we assess if the expected value and the amount/variance influence individual behavior. In practice, some questions present the same choice except for the expected value that can be positive, negative or zero, and some questions present the same probability for the options which are different only for the stake size. Based on the answer to these questions, we try to classify people in groups by means of multivariate analyses.

Second, following Deck and Schlesinger [2014] and ?, we try to identify "mixed risk averse" and 
"mixed risk loving" behavioral patterns (see Appendix B.2 for a terminological discussion of these terms). In particular, we relate the behavior of people on different moments to see its consistency and we build a "financial all moments risk aversion score" based on Scott and Horvath [1980] theoretical prediction.

Third, we build a "Rationality" score to see if there is substantial change in the results if we delete the less "rational" persons from the sample. However, our analysis is not on rationality but on the relevance of higher moments of the return distribution, that is, on the second of the assumptions listed at the beginning of this section. Indeed, even if some behaviors found in the behavioral finance literature (such as "anchoring", "overconfidence", "framing" ...: for a summary of these effects see De Bondt et al., 2008) can be almost surely defined as "irrational", many others can be seen either as "irrational" behavior or as "rational" behavior of agents with some kinds of "non-basic" utility function. For instance, Horvath and Sinha [2017] explain that "weighing losses (loss aversion and myopic loss aversion) more than gains of similar magnitude to be a consequence of rational decision". The debate about rationality overcomes the purpos e of this paper.

Finally, the work provides an additional investigation to check whether risky decisions are correlated with particular personal features. For instance, Halek and Eisenhauer [2001] investigate the correlation between risk aversion and some demographic characteristics like age, employment and education, finding that such characteristics strongly influence risk aversion.

Even if, in the recent literature, there are some experiments analysing the $n-t h$ degree risk preferences, we contribute to this literature for many reasons, besides the already exposed large number of analyses performed. First of all, we analyse the behavior with a short questionnaire built as simply as possible. Indeed, our 14 questions are binary choices between two alternatives composed of binary risks (that is with only two possible outcomes) till the third moment. As proved by Ebert [2015] (starting from Chiu, 2010), easily understood binary risks are enough to elicit risk preferences up to the third moment, that is, they are able to build two alternatives with the same mean and variance but different skewness. Moreover, we try to be as simple as possible also in the analysis of fourth moment preferences, with only 3 or 4 equiprobable outcomes. We stop the analysis at the first four moments, because Deck and Schlesinger [2014] study also the fi fth and sixth moments finding that behavior at these orders is only marginally different from making random choices. They affirm: "although we can theoretically consider risk preferences for any arbitrary order $\mathrm{n}$, restricting any analyses within economic applications to only the first four orders seems a reasonable approximation. We attribute this phenomenon to the ever increasing complexity involved with deciphering higher degrees of risk increases."

Secondly, we collect results from a very heterogeneous population, while previous studies usually use smaller samples or samples composed only of students: for instance, Deck and Schlesinger [2010] use a sample of 99 students, Ebert and Wiesen [2011] 72 students, Maier and R $\tilde{A} \frac{1}{4}$ ger [2012] 72 participants, Deck and Schlesinger [2014] 150 participants with a majority of students, Ebert [2015] 63 students, Deck and Schlesinger [2016] 53 participants, Bleichrodt and van Bruggen [2018] 122 participants. Indeed, to the best of our knowledge, only Noussair et al. [2014] and ? perform an analysis on a large and diversified sample. Compared to these two papers, our analysis has an added value derived from the dimension as well as the heterogeneity of respondents. ? analysed a smaller sample including respondents only from China, Germany and USA, and some analyses rely on a small subsample, while Noussair et al. [2014] solely consider Dutch people. Instead, by using the powerful tool of Amazon Mechanical Turk, our dataset includes people from different countries and with heterogeneous socio-demographic characteristics. By having a large and heterogeneous sample we manage to: (i) provide more general and unbiased results, (ii) analyse the different risk attitudes among countries and geographical areas. The latter topic is still scarcely empirically in- 
vestigated, even if it could be relevant. Indeed, ?, ? and ? provide evidence of differences in risk attitudes across countries, and ? find that Chinese people are a little more risk prone compared to US and German people. However, the geographical difference is not the only relevant feature: many papers find that women are significantly more risk-averse than men (see, for instance, ?, ?; ?, ?; ?, ?; Ebert and Wiesen, 2014; Noussair et al., 2014). Moreover, Ebert and Wiesen [2014] find that, with marginal significance, women are also more prudent and temperate, and Noussair et al. [2014] find that females are more temperate, but they find no gender effect for prudence. Noussair et al. [2014] report also some other very interesting results, such as older people are less risk averse, and people with high cognitive ability and high education are more prudent. such as smoking and heavy drinking. In our paper, we also correlate decisions on lotteries with non financial risk-related behaviors.

In addition, given that we collect part of our data by implementing a standard laboratory experiment, as a corollary, this work is useful to prove the external validity of data gathered in such a way.

The rest of the paper is structured as follows: before starting the experiment description, Section 2 reports a brief literature review. Section 3 explains the experiment and the scores built to perform the subsequent analyses. Section 4 reports the main results and, in particular, Section 4.1 reports the results on the questions regarding choices with different second moments of the return distribution, Section 4.2 regards the third moment, Section 4.3 regards the fourth moment, while Section 4.4 regards the overall pattern and Section 4.5 summarizes the results. Section 5 concerns the sociodemographic characteristics of subjects that can influence the financial risk aversion ${ }^{1}$. Finally, Section 6 concludes. In Appendix A we list all the questions of the Questionnaire. In Appendix $\mathrm{B}$ we present two very relevant discussions, useful for the whole paper, on a methodological issue (explaining that the risk apportionment task is restrictive and relatively complex) and on some terminological issues. Finally, Appendices C-D-E contain an in-depth analysis of the study reported in Section 4.1-4.2-4.3 respectively, including many analyses based on the joint distribution of replies to more than one question.

\section{Literature review}

Most of the decisions we face have uncertain outcomes and, for this reason, risk aversion is one of the issues that has drawn a lot of interest among economists and psychologists. The pioneering work on the St. Petersburg Paradox led to extensive research devoted to understanding decision under uncertainty. The first and widely accepted theory to formalize and explain this kind of decision is the Expected Utility Theory (EU, hereafter) axiomatized by ?. This theory is based on a set of assumptions useful to define rational behavior in a context with different possible outcomes whose probabilities are known and correspond to the objective ones. This theory was successful since it is mathematically easy to manage and, thanks to its assumptions, it allows to represent preferences with a utility function. In this framework the direct measure for risk aversion is the concavity of the utility function, i.e., a negative second derivative. This idea was also formalized by Pratt [1964] who defines both a relative and absolute measure of risk aversion based on the ratio between the second and the first derivative of the utility function.

In the early 50s, Allais [1953] demonstrates the fallacy of the assumptions at the root of the EU

\footnotetext{
${ }^{1}$ In the paper we will often include "risk aversion", "prudence" and "temperance" in the general risk aversion concept. In other words, we will use the term "risk aversion" some times with the stricter meaning of preference for a lower variance, and sometimes with the extended meaning of "all moments risk aversion" behavior (usually called "mixed risk aversion" in the literature) explained in Appendix B.2. Indeed, as explained in Section 2, Scott and Horvath [1980] prove that risk aversion should theoretically imply the "all moments risk aversion" behavior.
} 
theory. Indeed, subjects seem to make choices that especially violate the independence axiom. Based on this result, different theories emerge. The earliest contribution is the well-known Prospect Theory (Tversky and Kahneman, 1979) whose main pillars are: $(i)$ when taking decisions, subjects make their evaluations based on a neutral reference point; (ii) subjects are loss averse: losses appear "larger" than gains; (iii) the value function replaces the standard utility functions: such function is concave in the domain of gains and convex for losses; $(i v)$ the probability weighting function replaces the objective probabilities usually implemented in the EU: in taking decisions subjects usually overweight small probabilities and underweight high probabilities.

On the same strand, other theories try to explain the inconsistency of individual decisions with the cognitive distortion in evaluating probability or outcomes. Some examples are: cumulative prospect theory (Tversky and Kahneman, 1992), rank dependent expected utility (Quiggin, 1982; Quiggin, 1991), regret theory (Loomes and Sugden, 1982), maxmin expected utility (Gilboa and Schmeidler, 1989), just to name a few. Close to these theories, Prelec and Loewenstein [1991] find evidence for what they call "peanuts-effect": subjects, who in general prefer to avoid risk, are shown to be less risk averse when playing for "peanuts", meaning for a small amount of money. In this case, we can conclude that individual risk preference changes according to the amount of money they are betting for. All the above-mentioned arguments represent the bedrock of what we currently call behavioral economics. The main peculiarity of this approach is to explain anomalies or, more in general, individual choice based on psychological explanations.

In the same years, the analysis of optimal portfolio decision led to the emergence of a new strand of literature rooted in the importance of the higher moments of returns distribution. Authors like Samuelson [1970] and Tsiang [1972] trace back the main flaw of typical mean-variance approach (Markowitz, 1952) to the evidence that they only take into account the first and the second moment of the returns distribution. Indeed, considering only the first and second moment of the distribution leads to approximate solutions. For instance, the evidence proposed in many papers (such as Samuelson, 1970; Kraus and Litzenberger, 1976; Scott and Horvath, 1980; Kane, 1982) shows the existence of the so-called preference for (positive) skewness. Therefore, in the framing of the meanvariance theory, choices favoring options with positive skew, high risk and low expected return are interpreted as risk loving behavior, while, according to what Scott and Horvath [1980] suggest, it can be the rational behaviour of a risk averse subject that prefers a return distribution with higher odd central moments and lower even central moments. Indeed, Scott and Horvath [1980] prove that, starting from the standard assumptions of a utility function with positive first derivative (positive marginal utility) and negative second derivative (risk aversion), a subject will prefer higher values of the odd moments and lower values of the even moments of the return distribution. Formally

$$
(-1)^{n} u^{n}(x)<0 \quad \forall 0 \leq x \leq 1
$$

where $u^{n}$ is the n-th derivative of the utility function. According to Ekern [1980], a subject with this alternating sign of the derivatives can be defined as $n$-th degree risk averse. In the mean-variance standard framework only the positive first and the negative second derivative have been considered and labeled as non-satiation and risk aversion, respectively. The positive third, the negative forth, and the positive fifth derivatives were christened subsequently as prudence (Kimball, 1990), temperance (?, ?), and edginess (Lajeri-Chaherli, 2004) respectively.

If many experiments with the goal of testing the goodness of the above-mentioned alternative theories such as Prospect Theory (see, among others, Hey and Orme, 1994; Thaler et al., 1997; Gneezy and Potters, 1997) or the so-called "peanuts-effect" (see Weber and Chapman, 2005, and Green et al., 1999) have been run, the idea of preference for higher moments of the return distribution has remained untested for a long time. The main reason is the arduousness of measuring it 
with a simple test. Even if the empirical analysis seems to be still at the outset, after the paper by Eeckhoudt and Schlesinger [2006], in the last few years a fair number of experimental investigations have been performed on this topic (for a comprehensive review see Gollier et al., 2013, and Trautmann and van de Kuilen, 2018). The milestone study of Eeckhoudt and Schlesinger [2006] implements a model-independent way to study higher-order risk attitudes, and coin the terminology "risk apportionment of order n". However, all the studies that use the risk apportionment methodology restrict the analysis to lotteries that represent only a specific way of building comparisons on the higher moments, as explained in Appendix B.

Now, we report some of the results found in the previous literature. Astebro et al. [2009], implementing a sequence of pairwise choices similar to Holt and Laury [2002], discover that subjects are more willing to make risky choices if they face a positive skewed distribution of returns. This could be classified as evidence for prudence even if, in a subsequent work (Astebro et al., 2015) the authors address this behavior to an overweighting effect, meaning that the results they obtain are explained by using a specific probability weighting function. Deck and Schlesinger [2010] implement an experiment with pairwise choices among multi-armed lotteries testing for both prudence and temperance. What they find is that subjects made choices that could be classified as prudent but intemperate, meaning that they prefer to add a zero-mean risky option to the highest outcome but, at the same time, they prefer a lottery with just one option with a compounded risk. ? propose to a sample of students to make choices for 16 pairwise lotteries. The main goal of the experiment was to test for prudence, while no lottery for testing kurtosis was implemented. They find that only almost $50 \%$ of participants made prudent choices. Noussair et al. [2014] investigate the preference for both prudence and temperance in a very large representative sample of Dutch population. The results they obtain show a correlation between risk aversion, prudence and temperance: the majority of people prefers safe, prudent and temperate alternatives.

To sum up, the evidence that subjects have preference for skewness is robust: besides the above mentioned studies, other works such as Deck and Schlesinger [2014], ? and Baillon et al. [2018] observe that the majority of people make prudent choices. Regarding the role played by the fourth moment, results are not so strong: it has been shown that the proportion of people making temperate choices is usually smaller than that making prudent ones (see, for example, Ebert and Wiesen, 2014; Heinrich and Mayrhofer, 2018; and Krieger and Mayrhofer, 2017). For a quick resume, see Appendix A1 of ?.

Lastly, in this paper we follow the model-independent literature strand and we will not address the topic of confirming or not some theoretical theories. For instance, on one hand, all of the commonly used utility functions exhibit "skewness preference" but, on the other hand, as explained by Ebert [2015], "success of decision theories like cumulative prospect theory (Tversky and Kahneman, 1992) may be attributed in part to the fact that they imply skewness preference". Therefore, which theories are compatible with the features found in our experiment will not be the main focus of our discussions.

\section{The experiment}

\subsection{Experimental Design}

For our experiment, we build a small-medium size questionnaire composed of 14 choices regarding financial risk, 9 questions on non-financial risks, and 11 personal questions. All the questions are reported in Appendix A. To avoid any bias due to the order of appearance, we randomize the order of the financial risk block queries, so that each subject answers the same questions but following a 
different order. We try to avoid a lengthy task and questions are presented in a very simple way, that is they are binary choices and each choice presents a binary risk till the third moment analysis. Indeed, as explained by Ebert [2015], it is possible to provide an explicit re-parametrization of binary risks in terms of their first three moments. Therefore, about half of the respondents take less than 7 minutes and the average time is about 10 minutes.

The questionnaire on the financial risk part is composed of:

- 1 question on first moment (Question "zero");

- You have the possibility to participate in a lottery in which you have $50 \%$ probability of winning $100 \$$ and $50 \%$ probability of winning nothing. There is no participation fee. By choosing Option A you participate in the lottery, otherwise by selecting Option B you do not participate. Which option do you choose?

- 7 questions on second moment (risk aversion);

- Example: You have the possibility to choose between two hypothetical options. Option A: you have a $50 \%$ probability of winning either $5,000 \$$ or $15,000 \$$, Option B offers you a sure gain of 10,000\$. Which option do you choose?

- 4 questions on third moment (prudence);

- Example: You have the possibility to choose between two hypothetical options. Option A: you have a chance in a million to win a million Dollars and 0 otherwise. Option B: gain $1 \$$ for sure. Which option do you choose?

- 2 questions on fourth moment (temperance).

- Example: You are asked to choose between two hypothetical options with multiple outcomes. Option A: there is the possibility to gain, with the same chance equal to $33 \%$, $0 \$$ or 5,000\$ or 10,000\$. Option B: there is the possibility to gain, with the same probability of $25 \%$, $0 \$$ or 2,100\$ or $7,900 \$$ or $10,000 \$$. Which option do you choose?

Moreover, we add a consistency question ${ }^{2}$, that is the age ten years ago, and we build a rationality score (see subsection 3.3); subsequently, we delete two subjects who were both inconsistent and with a rationality score equal to zero, assuming that all answers were randomly chosen. We delete also two respondents with missing answers. At the end, the dataset includes 478 subjects. The experiment involves a large and heterogeneous sample. We run the experiment both with a standard tool, i.e. using laboratory experiment, and the innovative Amazon Mechanical Turk (AMT) tool. AMT is an integrated participant compensation system able to obtain data inexpensively and rapidly. This tool is a particularly powerful method to obtain high-quality data, as explained by Buhrmester et al. [2011]. The sample is, therefore, composed of two sub-samples:

- 83 students from University of Macerata who perform a laboratory experiment;

- 395 respondents from Amazon Mechanical Turk.

The comparison between the two sub-samples shows that results are consistent despite the fact that the second sub-sample is much more demographically diversified and is not directly controlled as a lab experiment is. For this reason we will present the analysis as if we had a unique sample composed of 478 subjects. Moreover, deleting a larger number of respondents (all the "inconsistent"

\footnotetext{
${ }^{2}$ We also insert a further control question similar to a captcha, meaning that we ask to report an alphanumerical code shown in a picture. In addition, we check for the response time and we do not pay subjects who took less than 2 minutes to answer to the whole questionnaire.
} 
persons, or those who answer B to Question "zero", or all subjects with a rationality score below 2 or 3 ) the results are almost unaffected showing a strong robustness.

Table 1 reports the two options proposed in each question with the corresponding expected value, standard deviation (the square root of the second central moment), third and fourth central moments and percentage of choices.

\subsection{Payment structure}

A related issue concerns the payment structure. We decide to give a small fixed amount to all respondents (excluding those who take less than 2 minutes to answer the whole questionnaire), without relating the amount to the answer of one or more questions. On one hand, this way reduce the incentive to give the "correct" answer. However, von Gaudecker et al. [2011] and Noussair et al. [2014] find that hypothetical lottery questions are a valid, unbiased, instrument to elicit risk attitudes: for instance Noussair et al. [2014] find no significant differences between the real and the hypothetical treatments, suggesting that "non-incentivized choices provide unbiased estimates of the average attitudes of a population for similar real stakes".

On the other hand, we avoid two kind of biases. The first is the standard bias due to the fact that experiments usually yield real gains but not real losses (see Maier and R $\tilde{4} \frac{1}{4} g e r, 2012$ ). The second bias is related to the stake size. Indeed, given the limited budget, the vast majority of the experiments regards choices with small amounts and with few respondents. As we will observe in our analysis, small amounts and large amounts can imply different behavior, as proposed by some theories, such as the already cited "peanuts-effect", or by authors such as Eeckhoudt and Schlesinger (2006), who suggest that individuals might be more likely to disaggregate risks ("good with bad" 3 ) if they are large, and to aggregate risks ("bad with bad") if one of the risks is relatively small. This result is confirmed by Deck and Schlesinger [2010] who find a (small) increase of prudence and an increase of temperance (even if the majority of their respondents presents an intemperate behavior) with the growth of the stake size, by Noussair et al. [2014] who find an increasing risk aversion and temperance (not prudence), and by ? who find an increasing risk aversion ${ }^{4}$. Moreover, Noussair et al. [2014] explain that they use stakes of the magnitude of approximately one year of after-tax incom e for the median participant, but it would be interesting to also consider decisions made over stakes that correspond to lifetime earnings, as done in the survey questions of ?. Therefore, we think it is important to ask questions with very high amounts (for instance, our Question 7) and it is not compatible with a real payment (unless the amounts are rescaled with the consequent distortion of the results if the mechanism is known by respondents). Moreover, the large number of respondents could produce a better and less biased output, as explained in the Introduction.

\section{3 "Rationality" score and Question "zero"}

As already explained, we build a "rationality" score that can be used either to select/delete subjects from the sample or to study if the behavior changes for different value of "rationality" . This score assumes values from 0 (totally "irrational") to 5 (completely "rational"), with three components.

\footnotetext{
${ }^{3}$ See Appendix B.1 for the definition of prudence, temperance and risk apportionment of higher orders based on the combination of "good with bad" or "good with good" and "bad with bad".

${ }^{4}$ They also find a lower percentage of risky decisions also for orders from 3 to 6 , especially for even orders, but these differences are not statistically significant. However, we think that this result could be driven by two reasons: first, only 48 persons are involved in this part of the analysis; second, the compound lotteries could present the already explained growing complexity for increasing orders, that induces a growing number of random answers.

${ }^{5}$ Noussair et al. [2014] perform something similar: they include in the laboratory experimental session the Frederick's (2005) cognitive reflection test to measure the cognitive ability of students. We prefer to deduce the cognitive ability from the main questionnaire in order to keep the experiment as simple and short as possible.
} 


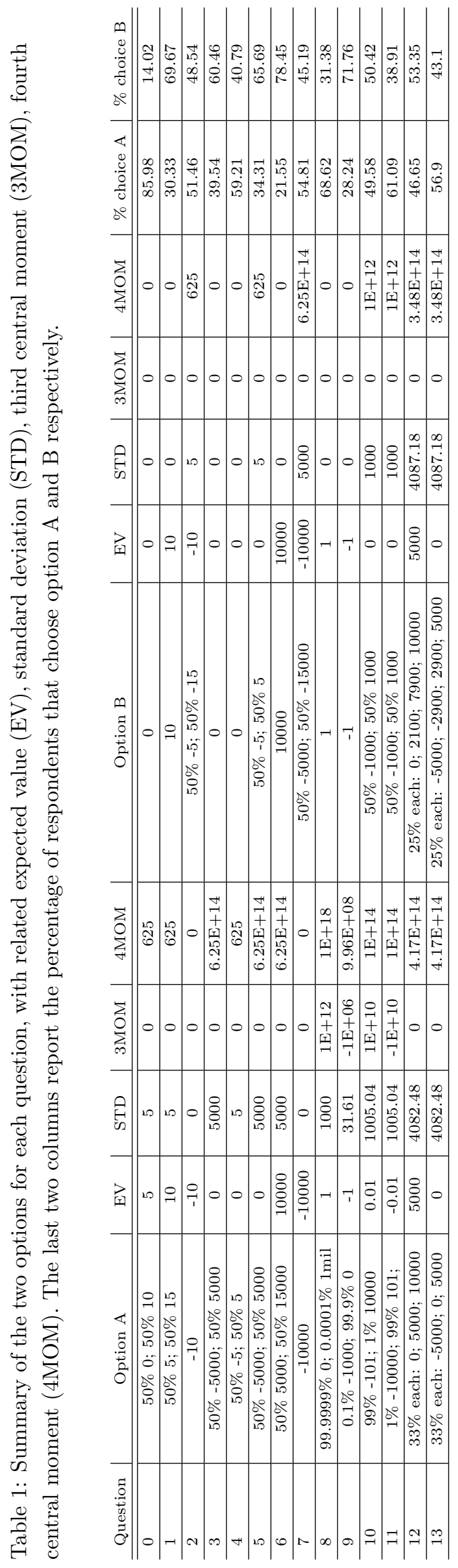


Table 2: "Rationality" score distribution.

\begin{tabular}{|l|c|c|c|c|c|c|c|}
\hline "Rationality" score & 0 & 1 & 2 & 3 & 4 & 5 & Total \\
\hline Frequency & 3 & 17 & 39 & 96 & 167 & 156 & 478 \\
\hline Percentage & 0.63 & 3.56 & 8.16 & 20.08 & 34.94 & 32.64 & 100 \\
\hline Cumulative percentage & 0.63 & 4.18 & 12.34 & 32.43 & 67.36 & 100 & \\
\hline
\end{tabular}

The first part is related to the question on the first moment, that we label Question 0. We assign 2 points for the choice of Option A and zero otherwise:

- option A: gain either $100 \$$ or $0 \$$ with $50 \%$ probability;

- option B: do not participate the lottery.

The choice of option A should be straightforward because the expected value of option A is higher and the worst case of option A is exactly the sure value of option B: in other words, choosing option A you can win or not, while choosing option B you are sure not to win. Nevertheless, $14 \%$ of respondents (both in the AMT and in the University of Macerata samples) choose option B. We try to observe if these respondents were absolutely risk averse in the other questions both on financial and non-financial risks, as a proxy for people who could apply moral arguments against gambling, but we do not find this confirmation: for instance, the correlation between this choice and the "financial all moments risk aversion score" (see Section 3.4) is -0.0178, not statistically significant (moreover, if it was significant, it would be negative, that is, the probability of choosing $\mathrm{B}$ reduces when a person is more risk averse). Therefore we classify this choice as "irrational" a nd we assign it 0 points.

The second part of the score depends on the options chosen in Questions 1, 2 and 4 (see Appendix A or Section C.1). These questions propose the same pairwise choice and they differ only in terms of expected value. In building "rationality score", we assume that subjects who show two preference reversals between the risky choice and the safe choice behave in an inconsistent way, so they are classified as "irrational". Therefore, to be more precise, we assign 0 points to subjects who choose 1A-2B-4B (that is, they choose option A in Question 1, option B in Question 2, and option B in Question 4) or 1B-2A-4A. Conversely, subjects who change their preference at most once, get 1 point.

Finally, the last part of the score is based on the transitivity property (if $\mathrm{X}$ is preferred to $\mathrm{Y}$ and $\mathrm{Y}$ is preferred to Z, X has to be preferred to Z), focusing on Questions 3, 4 and 5 (see Appendix A or Section C.3.1). These questions give the possibility of taking part or not in a lottery with zero expected value; the difference is in the second moment, and Question 5 is built comparing the risky options proposed in Questions 3 and 4. Subjects who always choose Option A or always choose Option B in Question 3-4-5 obtain 2 points (consistency of choices and preference monotonically increasing or decreasing with the lottery amount), subjects who choose $3 \mathrm{~A}-4 \mathrm{~B}-5 \mathrm{~B}$ or $3 \mathrm{~B}-4 \mathrm{~A}-5 \mathrm{~A}$ obtains 0 points (inconsistency of choices), and the other subjects receive 1 point.

To sum up, the "Rationality" score is the sum of points collected in the three described parts. Table 2 reports the number of respondents for each level of the score.

The results show that the vast majority of respondents obtain a "Rationality" score at least equal to 3. It partially explains why the results present very small changes when deleting the subjects with rationality score below 3, or deleting who answers B to Question "zero", or deleting inconsistent in the age question. 
Table 3: "Financial risk scores": options that obtain 1 point each (Questions with "X" are not used for the computation of the score.

\begin{tabular}{|l|c|c|c|c|c|c|c|c|c|c|c|c|c|}
\hline Question & 1 & 2 & 3 & 4 & 5 & 6 & 7 & 8 & 9 & 10 & 11 & 12 & 13 \\
\hline Risk averse option & B & A & B & B & B & B & A & X & B & X & B & B & B \\
\hline Alternate propensity option & A & B & A & A & A & A & B & A & X & A & X & A & A \\
\hline
\end{tabular}

\subsection{Mixed risk aversion and love}

One of the goals of this paper, as explained in Section 1, is to use the questions on financial risk in order to build a "financial all moments risk aversion score" based on Scott and Horvath's (1980) theoretical prediction. This is exactly the same as counting the number of risk averse or risk loving choices performed by Deck and Schlesinger [2014] and ? to assess the presence of a "mixed risk averse" behavioral pattern (Appendix B.2 presents an in-depth discussion of the meaning and of the problems of the terminology used in this section). Moreover, given that the cited papers also search for "mixed risk loving" behaviors, we also build a "financial alternate propensity with even moments risk love score".

In particular, we build three scores on second, third and fourth moments and we compute the "financial all moments risk aversion score" (mixed risk aversion) as the sum of the three scores.

The score for the second moment is computed in the following way: for each question we assign value 1 to those subjects who choose the option with the smaller second moment and 0 otherwise. Since we propose 7 questions, the second moment score ranges from 0 to 7 .

In a similar way, the score for the third moment is computed by assigning 1 point to subjects who choose the option with the larger third moment in Question 9 and 11 and zero otherwise. We exclude Questions 8 and 10 because they contain a "struggle" between different moments, that can take risk averse people to choose both alternatives. In this case, since we are taking into account only two questions, the score ranges from 0 to 2 .

Lastly, for the fourth moment score, respondents that choose the option with smaller fourth moment in Questions 12 and 13 obtain one point and zero otherwise, with a final score that ranges from 0 to 2 .

As already said, the total "financial all moments risk aversion score" is the sum of the three scores just described and, therefore, it ranges from 0 (very risk prone subject) to 11 (very risk averse subject). The row beginning with "Risk averse option" in Table 3 shows, for each question, the choice that a risk averse subject would have made. Subjects who follow this path of answers, obtain 11 points for the "financial all moments risk aversion score" (obviously, the other option is the risk prone choice and obtains zero).

The "financial alternate propensity with even moments risk love score" (mixed risk love) is built following the same method and the choices are reported in the "Alternate propensity option" row of Table 3. Obviously, the choices are the opposite for the second and fourth moments. Instead, concerning the third moment, there is a "struggle" between even and odd moments for Questions 9 and 11 that are excluded, while we assign 1 point to subjects who choose the option with the larger third moment in Question 8 and 10.

\section{Main results}

We will summarize the main results in the following subsections. However, Appendices C-D-E report an extension of the analysis presented in subsections 4.1-4.2-4.3, including many analyses 
based on the joint distribution of replies to more than one question.

\subsection{Results on second moment analyses}

As already mentioned, we perform 7 questions on the second moment of the distribution of the returns, that is, on the so-called "risk propensity". These questions can be combined in different analyses in order to discover possible behavioral patterns and regularities.

The first analysis concerns Questions 1, 2 and 4, that present two options with the same expected value, but a different second moment even if the gap on the second moment is small: an option with a small variance and an even safer option with zero variance. The three questions differ for the expected value: Question 1 has a small positive expected value, Question 2 presents a small negative expected value and Question 4 has zero expected value. Table 1 reports both the options and the results. When the expected value is positive, people usually prefer the sure gain (almost 70\% of respondents chooses answer B in Question 1), that is there is risk aversion (second moment/variance aversion). Instead, when the expected value is negative, preferences between a sure loss or a variable loss are equally divided, indeed the answers in Question 2 are not statistically different from a $50 \%-50 \%$ division. Lastly, when the expected value is zero, people often decide to participate in the lottery (59\%) showing a risk prone behavior. Therefore, when the gap in the second moment between the two options is low, we show a huge heterogeneity in behaviors with quite different responses to a second moment increase over gains, losses and when the expected value is zero, that can not be caught by any proposed theory. This result is in line with von Gaudecker et al. [2011] who state: "the large preference heterogeneity that we document is likely to stand behind the mixed evidence accumul ated so far". The whole analysis on Questions 1, 2 and 4 is reported in Appendix C.1.

The second analysis concerns Questions 3, 6 and 7, that present two options with the same expected value and a large gap between the second moment of the two options. All questions have the same variance gap between the two options. However, they present three different expected values: Question 3 has zero expected value, while Question 6 has a positive expected value, and Question 7 has a negative one. As shown by Table 1, the vast majority of people is risk averse when the lottery has a positive expected value: almost $80 \%$ of subjects answer B to Question 6, that is they prefer the sure gain. People tend to be risk averse also when the lottery has zero or a negative expected value, but in this case there is a much larger percentage of subjects that choose the risky option: $40 \%$ and $45 \%$ or respondents respectively. However, the percentage of people who choose the safe option is above and statistically different from $50 \%$ even when the expected value is negative (54.81\% in Question 7). Therefore, when the gap between the second moments of the two options is high, a lot of people are consistently risk averse irregardless of the expected value, but there is quite a large group of subjects that is risk averse when the expected value is positive and is risk prone in losses, while consistently risk prone subjects are almost nonexistent. For a deeper analysis on Questions 1, 2 and 4, see Appendix C.2.

The third analysis concerns Questions 3,4 and 5, in order to study the different behavior in presence of different (low and high) second moments gaps between the two options, when the expected value of the options is zero. Question 4 presents a low gap between the variance of the two choices, while Questions 3 and 5 have a larger variance gap between the two options, that is the bet in Questions 3 and 5 present larger amounts. Between Questions 3 and 5 the difference is that Question 3 presents a sure choice, while both choices in Question 5 have a positive second moment. The majority of people tend to be risk prone when the lottery is for a low amount ( $59 \%$ of answer A in Question 4), but Question 3 and 5 show that they become risk averse when the stake is large 
(60\% and $66 \%$ respectively). Therefore, the value (or the gap between the two options) of the second moment is an important determinant of the choice because, beside the two groups of risk averse and risk prone persons, there is another large group of subjects who are risk prone for a low second moment and risk averse for a large one. The whole analysis on Questions 3, 4 and 5 is reported in Appendix C.3.1.

The last analysis on the second moment compare both Questions 1-6 and 2-7 to check whether there are significant differences in subjects' behavior when the expected value is either positive or negative. Questions 1 and 6 present two options with positive expected value, but different second moments. Among the two questions, Question 1 presents a low expected value and a low gap between the variance of the two options, while Questions 6 presents a high expected value and a large gap between the two options. The same holds for Question 2 and 7, but for the expected values that is negative in both questions. The first result is that risk aversion increases when the second moment gap increases: a larger percentage of respondents chooses the safe choice comparing Question 6 with Question 1 and Question 7 with Question 2. Moreover, people are usually consistently risk averse over gains, while when the expected value is negative people are very heterogeneous. To summarize, we can state that: (i) risk aversion increases when the second moment increases; (ii) subjects are usually risk averse in gain; (iii) there is high heterogeneity in behaviors when the expected value is negative. For a deeper analysis on Questions 1-6 and 2-7, see Appendix C.3.2.

\subsection{Results on third moment analyses}

In this section, we focus on the behavior of our respondents to choices that involve a different third moment of the distribution of returns (and different other moments too), always keeping the same expected value between the two options.

We start our analysis with Question 10: both options have the same first and second moments, but Option A presents larger higher order moments. It implies that, as predicted by Scott and Horvath (1980), a risk averse person should prefer Option B because of its lower even moments, but should prefer Option A because of its higher odd moments. Option A has the "third moment robust risk aversion" feature ("kurtosis robustness feature of prudence", using the definition of Ebert, 2013b, as explained in Appendix B.2), that is the preference for a higher third moment even in presence of a higher fourth moment (and higher even moments of higher order); instead, Option B has the "fourth moment very robust risk aversion" feature, that is the preference for a lower fourth moment even in presence of a lower third moment. The results reported in Table 1 show that the sample is almost equally divided (not statistically different from a random choice), confirming the "struggle" between two opposite effects without a prevalence.

Questions 8 presents two options with the same expected value, but Option A presents larger higher order moments (including the second moment). As for Question 10, as predicted by Scott and Horvath (1980), a risk averse subject should prefer lower even moments (option B) and higher odd moments (option A). Option A has the "third moment very robust risk aversion" feature (third moment even more important than second one), while Option B has the "second moment robust risk aversion" feature.

In this case, the result shows a strong result: almost the $70 \%$ of respondents prefer Option A, that is they show the "third moment very robust risk aversion". The reason for the different behavior between Questions 8 and 10, could be the gap between the third moment of the two options, which is much larger in Question 8 compared to Question 10. In other words the third (odd) moment could be more relevant even than the second (even) moment, confirming the papers that find support for prudence (Astebro et al., 2009; Deck and Schlesinger, 2010, 2016; Ebert and Wiesen, 2011, 2014; 
Maier and Ruger, 2011; Noussair et al., 2014), but the gap between the third moments of the two should exceed a very significant threshold.

To sum up, in presence of positive odd higher moments, we find that the majority of the people presents the "third moment very robust risk aversion" when there is a strong gap in the value of the third moment, that is the third (odd) moment seems even more relevant than the second (even) moment (confirming literature findings about the importance of "prudence"). With a smaller third moment gap, almost half of the people that show the "third moment very robust risk aversion", do not present it anymore and they do not even show "third moment robust risk aversion". For a deeper analysis on Questions 8 and 10, that is questions with positive odd higher moments, see Appendix D.1.

Now, we study questions with negative odd higher moments. We start with Question 11: both options have the same first and second moments, but Option A presents larger negative higher order odd moments and larger positive higher order even moments. In this case, as predicted by Scott and Horvath [1980], a risk averse person should surely prefer Option B because of its higher odd moments (odd moments are zero because Option B is symmetrical, while they are negative in Option A) and lower even moments of the pay-off. Surprisingly, persons usually prefer Option A (more than $61 \%$ of respondents), as reported in Table 1.

Question 9, again, presents two choices with the same expected value but different higher order moments. Indeed, Option A presents larger negative higher order odd moments and larger positive higher order even moments (including the second moment). Following Scott and Horvath [1980], a risk averse subject should surely prefer Option B (higher odd moments and lower even moments). Table 1 shows a strong result: people usually tend to be "prudent", indeed $72 \%$ of respondents choose Option B.

Therefore, two opposite results emerge from the analysis of the two questions. A possible explanation is the presence of a higher second moment (and higher values of all orders even moments) in Option A of Question 9 that fosters the choice of Option B in this question, while the second moments are equal between the two options in Question 11; related to this explanation, we can add the fact that in Question 9 there is a "safe" option (sure pay-off, higher central moments equal to zero), while it is missing in Question 11, where both options have an uncertain pay-off. However, the surprising result of Question 11 should be further investigated in future experiments.

Summing up, in presence of losses (involving negative third and other odd higher moments), we observe a puzzling result of "unstable" behavior. The whole analysis on Questions 9 and 11 is reported in Appendix D.2. Moreover, Appendix D.3 includes a further analysis on the joint behavior of respondents about the two pairs of Questions 8-9 and 10-11.

\subsection{Results on forth moment analyses}

Focusing on Questions 12 and 13, we study choices between two options that present the same expected value, (almost) the same second moment and the same odd higher order moments (all equal to 0 , given that the return distributions are symmetric), but different fourth (and higher) even moments. Indeed, Option A presents larger higher order even moments in both Question 12 and 13. The difference is that Question 12 has a positive expected value (equal to $5000 \$$ ) and even in the worst scenarios there aren't losses, while Question 13 presents zero expected value with possible losses. Scott and Horvath (1980) predict that a risk averse person should prefer Option B because of its lower kurtosis, in both Questions.

The result is reported in Table 1. For Question 12, we can observe that, even if there is a preference for Option B as predicted, this percentage of $53.35 \%$ is statistically different from an equal $50 \%$ division at $10 \%$ of confidence level, but not at $5 \%$. Therefore, we can find a weak 
tendency to the "fourth moment risk aversion" (the so-called "temperance").

Questions 13 presents a choice with the same structure of Question 12, but the expected value is zero. In this case, the majority of people $(56.90 \%)$ prefer Option A, that is the option with higher kurtosis, against the Scott and Horvath [1980] prediction. Therefore, when the expected value is not positive, people often seem to show a preference for risk (no "temperance").

On one hand, these results are weak and similar to random choices, confirming the contrasting findings on "temperance", with values around 50\%-50\% especially if the lotteries are presented in a reduced form (Deck and Schlesinger, 2016, find $47 \%$ of temperate choices, and ?, ?, find 50\%). A possible explanation can be given by the increasing difficulty of the questions (an increasing number of possible outcomes) when the moment increases, that induces respondents to choose randomly one of the options.

However, on the other hand, we find a relevant (even if weak) result that should be further studied in future experiments: similarly to the second moment, there is a difference in the behavior when the expected value is positive or not. In particular, with a positive expected value, there is a tendency towards risk aversion ("temperance"), while with a zero expected value there is a tendency towards risk love. For a deeper analysis on Questions 12 and 13, see Appendix E.1.

Given that these results have some similarities with the results on the second moment, and given that the prediction of Scott and Horvath (1980) on higher moments is based on the preference on first and second moment, in Appendix E.2 we study jointly the behavior in questions regarding second and fourth moments. Here we briefly summarize the main results of this analysis. First of all, subjects focus more on the second moment rather than the fourth. However, in presence of gains (positive expected values), we are able to observe a slight tendency to find consistent choices on even moments as predicted by Scott and Horvath [1980] and, in particular, people often prefer lower even moments (they are consistently risk averse). Instead, in presence of a zero expected value, we do not find the tendency to be consistent and the majority of respondents tends to be risk prone. This behavior on the fourth moment seems very similar to the behavior on the second moment when the variance ga $\mathrm{p}$ of the two options is low, in a sort of "extended peanuts effect". A possible explanation is that the effect of the fourth moment can be compared to the effect of low gaps in the second moment. Another possibility is that the gap between the fourth moment of the two options presented in our questions is too low, while in the presence of a larger gap subjects could go back to a risk aversion ("temperance") to even moments as happened for the second moment. Further empirical studies could try to understand this feature.

\subsection{Mixed risk aversion and love: results}

As explained in Section 3.4, similarly to ?, we build:

- a "financial all moments risk aversion score" composed by three scores on second, third and fourth moments, and based on Scott and Horvath 's (1980) theoretical prediction. It assesses the presence of the so-called "mixed risk averse" behavioral pattern;

- a "financial alternate propensity with even moments risk love score" composed of three slightly different second, third and fourth moments scores, that assesses the presence of the so-called "mixed risk love" behavioral pattern.

Let's start with the "financial all moments risk aversion score". Figure 1 reports the distribution of respondents for the three scores computed on the second, third and fourth moments.

Panel (a) shows the distribution for the second moment: we can see a clear tendency to be risk averse, that is to search the option with the lower second moment. If we compare the two percentages 
obtained by the two opposite behaviors, that is score 0 compared with score 7 , score 1 compared with score 6 and so on, we always find that the risk averse group includes about $9 \%$ more of subjects then the correspondent risk prone group. Indeed, only three subjects are absolutely risk prone (score equal to 0 ) and less than $5 \%$ of respondents obtain a score equal to 0 or 1 . However, there is about a quarter of subjects $(27.2 \%)$ that presents a moderate tendency to be risk prone, obtaining 2 or 3. Instead, almost half of the people $(46.1 \%)$ are quite risk averse with a score equal to 4 or 5 , and almost another quarter $(22.1 \%)$ is strongly risk averse, obtaining a score of 6 or 7 .

Distributions of respondents by "financial risk aversion score" on third and fourth moments are reported in panel (b) and (c) of Figure 1 respectively. In these cases we have a less detailed description given that the score ranges between 0 and 2. However, regarding the third moment, there is statistically significant evidence that people tend to prefer the choice with the higher third moment: more than $29 \%$ obtains 2 , while only $18 \%$ obtains 0 .

Instead, analysing the distributions of respondents on the fourth moment, we do not find clear behavior between the preference for a lower $(26 \%$ of subjects score 2 ) or a higher (29\% of subjects score 0 ) fourth moment. Moreover, the distribution is statistically different from a random binomial (Chi-Squared test) with a $95 \%$ but not with a $99 \%$ confidence interval.

The theoretical prediction of Scott and Horvath [1980] for a correspondence among the risk aversion propensities of all the even moments and of all the odd moments is only slightly supported by the data: there is a positive correlation between second and fourth "financial risk aversion score", but it is quite low, that is equal to $12 \%$ as reported in Table 4 . This table also shows a low but positive correlation between second and third moments $(14 \%$, in line with Deck and Schlesinger, 2016), while the correlation between third and fourth moment is almost null, as found by Deck and Schlesinger [2016] and as theoretically expected. These low correlations imply a large heterogeneity of behavioral patterns.r instance, an subject could be risk averse and "prudent", but "i ntemperate", while another one could be risk prone, but "prudent" and "temperate" and so on, without a clear scheme able to reproduce the behavior of a majority of people). Probably, this feature implies that the utility function of many subjects can hardly be reduced to be function of one or two parameters only.

(a) Second moment

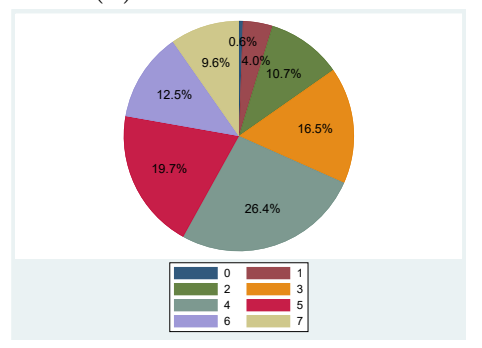

(b) Third moment

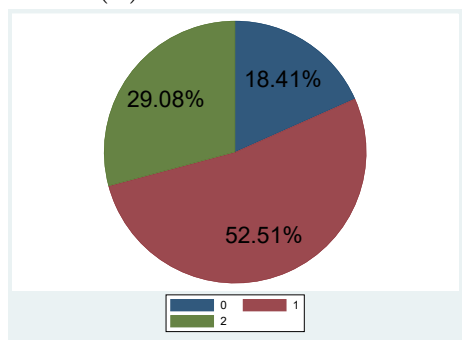

(c) Fourth moment

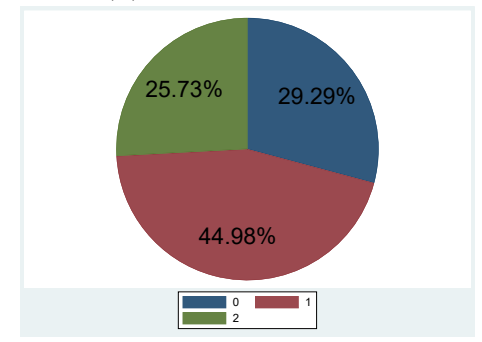

Figure 1: "Financial Risk Aversion score" on second, third and fourth moments.

The last column of Table 5 shows the distribution of subjects by total "financial all moments risk aversion score", that is the sum of the scores computed on the three moments. Given the tendency to be risk averse in the second moment, this distribution also shows a tendency of respondents for risk aversion. We can roughly divide the distribution in four buckets: risk prone people (scores 0-2) are very few (less than $4 \%$ and none obtains 0 ); moderately risk prone subjects (score 3-5) are almost a third of the sample; moderately risk averse subjects (score 6-8) are half of the sample, and very risk averse persons (score 9-11) are almost $15 \%$ of the sample. However, only two subjects are fully risk averse, while the vast majority of respondents, that is about $85 \%$, obtains intermediate 
Table 4: Correlations among second, third and fourth moments for "financial all moments risk aversion score" and "financial alternate propensity with even moments risk love score".

\begin{tabular}{|l|c|c|}
\hline All moments risk aversion & Moment 2 & Moment 3 \\
\hline Moment 3 & 0.14 & 1.00 \\
\hline Moment 4 & 0.12 & 0.03 \\
\hline
\end{tabular}

\begin{tabular}{|l|c|c|}
\hline Alternate propensity - even moments risk love & Moment 2 & Moment 3 \\
\hline Moment 3 & 0.05 & 1.00 \\
\hline Moment 4 & 0.12 & -0.01 \\
\hline
\end{tabular}

Table 5: Distribution of subjects by Financial Risk Aversion score (from 0 to 11).

\begin{tabular}{|c|c|c|c|c|c|c|c|c|c|c|c|c|}
\hline Risk Aversion score & 0 & 1 & 2 & 3 & 4 & 5 & 6 & 7 & 8 & 9 & 10 & 11 \\
\hline Number of respondents & 0 & 2 & 15 & 31 & 52 & 71 & 76 & 83 & 79 & 45 & 22 & 2 \\
\hline
\end{tabular}

values from 4 to 9 , showing they enjoy various levels of risk propensity.

As explained by Deck and Schlesinger [2014] making even one risk-loving decision might disqualify an individual from being labeled as "risk averse", therefore we will adopt a stochastic type of labeling, following also ? and Maier and RÃ $\frac{1}{4}$ ger [2012] (who explain that: "The fact that subjects of an experiment sometimes make different choices on the very same decision problem and under the same conditions is only reconcilable with stochastic theories"), and refer to someone who obtains 6 or more as being "risk averse". However, given that there could be "risk neutral" subjects, we also define a stronger measure, which labels as "all moment risk averse" (or "mixed risk averse") individuals who reach at least 7 or 8 in the "financial all moments risk aversion score".

We repeat the same analyses for the "financial alternate propensity with even moments risk love score". The second and fourth moment scores are the same, but we change the questions used for the third moment. Again, there is statistically significant evidence that people tend to prefer the choice with the higher third moment: $35.15 \%$ obtains 2 , while only $16.95 \%$ obtains 0 . Correlation between third and fourth moments is again not statistically significant but, in this case, also the correlation between second and third moment is very low (see panel (b) of Table 4), confirming the theoretical expected result of a lack of correspondence among the risk aversion between even and odd moments.

The distribution of subjects by total "financial alternate propensity with even moments risk love score", that is the sum of the scores computed on the three moments, is almost opposite to the "financial all moments risk aversion score". We divide the distribution in four buckets: people who score 0-2 points are $10.25 \%$, subjects who score 3-5 are about half of the sample $(50.42 \%)$, respondents who score 6-8 are $35.98 \%$, and people who largely display this behavior (score 9-11) are only $3.35 \%$ of the sample. We can refer to someone who obtains 6 or more as being a "financial alternate propensity with even moments risk love" subject (or "mixed risk lover"). However, it is better to define stronger measures, for individuals who make at least 7 or 8 in the score.

Table 6 shows the results of the labeling subjects as subjects with "financial all moments risk averse" and with "financial alternate propensity with even moments risk love". Using as a threshold 6, some respondents are classified as following both behaviors and it is theoretically not possible; we think that this classification is too weak. If we fix the threshold equal to 7 , almost half of our respondents can be classified as "financial all moments risk averse", while almost a quarter results as with "alternate propensity"; it is interesting to see that another quarter of respondents can not 
Table 6: "Financial all moments risk averse" subjects and "financial alternate propensity with even moments risk love" subjects.

\begin{tabular}{cc|ccc} 
& \multicolumn{4}{c}{ Alternate propensity (mixed risk love) } \\
\hline & Threshold $=6$ & 0 & 1 & Total \\
\hline All moments risk aversion & 0 & 4.60 & 31.17 & 35.77 \\
(mixed risk aversion) & 1 & 56.07 & 8.16 & 64.23 \\
& Total & 60.67 & 39.33 & 100 \\
\hline \hline & Threshold $=7$ & 0 & 1 & Total \\
\hline All moments risk aversion & 0 & 27.62 & 24.06 & 51.67 \\
(mixed risk aversion) & 1 & 48.33 & 0 & 48.33 \\
& Total & 75.94 & 24.06 & 100 \\
\hline \hline & Threshold $=8$ & 0 & 1 & Total \\
\hline All moments risk aversion & 0 & 58.16 & 10.88 & 69.04 \\
(mixed risk aversion) & 1 & 30.96 & 0 & 30.96 \\
& Total & 89.12 & 10.88 & 100 \\
\hline
\end{tabular}

be standardized in a behavioral pattern. The result is obviously enlarged when considering 8 as a threshold. ? find that $43 \%-62 \%$ (depending on the fixed threshold) of respondents are "mixed risk averter", and 8-14\% are "mixed risk lover" (similar to our result when threshold is 8). Even if the results are obviously driven by the num ber of questions on each moment and therefore can not really be compared, we partially confirm their result: (i) "financial all moments risk aversion" is the behavior of the largest group of the population, involving a much larger number of respondents compared to "alternate propensity" (even if we find that the number of "mixed risk averter" is twice or three times the amount of "mixed risk lover", while they find it is four or five times), (ii) a not negligible fraction of the population does not follow the two analysed behaviors.

\subsection{The overall picture}

In this section, we summarize our main results and compare them with the results found in the recent empirical literature and reported by ? in their Appendix A1.

1. The impact on the choices decreases when the order of the moment increases: for instance, all the other moments being equal, a small difference in the expected value has a much more relevant impact than a similar gap in the values of the higher moments. The increasing complexity of the choice could strengthen this behavior. However, this feature is surely true among even moments and among odd moments, but we are not sure that an even moment of order $n$ is more relevant than an odd moment of order $n+1$, indeed, odd moments seem to have a stronger impact than even moments ${ }^{6}$.

2. People usually prefer options with lower second moments. Overall, respondents choose the option with the lower second moment in questions from 1 to 7 in $60.19 \%$ of the cases. This result is in the range of the results found in this literature. Indeed, it is slightly below the percentage found in the experiments with compound lotteries (from $66 \%$ of Ebert and Wiesen,

\footnotetext{
${ }^{6} \mathrm{~A}$ word of caution is needed: we are inferring the relevance of the moment from the number of respondents that seem not to choose randomly, but this method could be wrong, because it does not perform a direct comparison of the relative strength of the different moments. To measure the intensity we should work with risk compensations (price list techniques) as done by Ebert and Wiesen [2014].
} 
2014, to $75 \%$ of ?, ?), but above $56 \%$ found in the experiment by Maier and RÃ $\frac{1}{4}$ ger [2012] performed with a reduced lottery.

3. The preference for a lower second moment is quite a common feature when the gap between the second moment of the two options is large and when the expected value is positive. When the gap is low and the expected value is zero or negative this preference seems to disappear and people present a large heterogeneity in behaviors.

4. People usually prefer a higher third moment: in questions from 8 to 11 , respondents choose this option in $57.22 \%$ of the cases. This result is in the range of the results found in this literature. Indeed, it is below the percentage found in the experiments with compound lotteries (from $61 \%$ of Deck and Schlesinger, 2010, to $89 \%$ of Noussair et al., 2014), but almost in line with the results found in the experiment performed with a reduced lottery $(56 \%$ by both Maier and R $\tilde{A} \frac{1}{4}$ ger, 2012, and ?, ?, while Deck and Schlesinger, 2016, found a larger 77\%).

5. The gap between the third moments of the two options is relevant. When it is very high, it can take respondents to choose the option with the better (higher) third moment even if it presents a worse (higher) second moment ("third moment very robust risk aversion" for some ranges of the gap of second and third moments).

6. However in presence of losses, that is a negative third moment (and other odd higher moments), we find "unstable" behavior. This puzzling result should be studied in future works.

7. People do not present a statistically significant preference for a higher or lower fourth moment: in questions 12 and 13, respondents choose the option with the lower fourth moment in the $48.23 \%$ of the cases. This percentage confirms the ambiguous findings of the previous experiments: with compound lotteries "temperate" choices are usually higher, even if there is also the very low $38 \%$ found by Deck and Schlesinger [2010], while with reduced lotteries results are similar (47\% found by Deck and Schlesinger, 2016; $50 \%$ by ?, ?; and $56 \%$ by Maier and RÃ $\frac{1}{4}$ ger, 2012). The result, not statistically different from a random choice, can be driven by both a weak preference and the difficulties due to the lotteries built to assess the fourth (and higher orders) moment.

8. Similarly to the second moment, there is a difference in the response to the fourth moment when the expected value is positive or not. In particular, with a positive expected value (gains), there is a tendency towards risk aversion, while with a zero expected value, there is a tendency towards risk love. Moreover, with a positive expected value, there is a tendency to perform consistent risk averse choices on even moments as predicted by Scott and Horvath (1980).

9. Subjects with a "financial all moments risk aversion" behavior ("mixed risk averter") are the largest group of the population, involving a much larger number of respondents compared to the "alternate propensity" behavior ("mixed risk lover"), but a consistent fraction of the population does not follow the two usually analysed behaviors. The large heterogeneity in behaviors is a recurrent feature, consistently with the findings of von Gaudecker et al. [2011]. Moreover, these authors also state that "while many people exhibit consistent choice patterns, some have very high error propensities", often associated with the propensity to choose randomly rather than on the basis of preferences, in line with our findings.

Beside some unexpected results that should be further investigated (see the results to Questions 4, 11 and 13), our experiment confirms the well established result of the preference of the majority 
of the respondents for higher odd and lower even moments, and highlights three features: (i) the importance of the gap level between the corresponding moments of the two choices is most relevant, (ii) the behavioral change in presence of a positive/zero/negative expected value has also to be considered, (iii) the complexity of the choice is a very important driver of the propensity to switch from choosing on the basis of preferences and choosing randomly to minimize the effort.

Denoting with $m_{j}^{i}$ the central moment of order $i$ of the option $j$, with $\Delta m^{i}=m_{A}^{i}-m_{B}^{i}$ the gap between the same moment of option $\mathrm{A}$ and option $\mathrm{B}$, with $O_{j}$ the possible outcomes of option $j$, assuming a utility function $U$ and denoting with $\Delta U$ the gap between the utility of Option $\mathrm{A}$ and the utility of Option B $\left(\Delta U=U_{A}-U_{B}\right)$, the largest group of the population, that is the "financial all moments risk averse" people, should choose Option A (in a stochastic way) if $\Delta U$ is positive and vice versa. $\Delta U$, for this group of people, is built in the following manner:

$$
\begin{array}{r}
\Delta U=f_{1}\left(\Delta m^{1}\right)-f_{2}\left(\Delta m^{2},\left|\Delta m^{2}\right|<\epsilon_{2}, \operatorname{sgn}\left(O_{A, B}\right)\right)+ \\
f_{3}\left(\Delta m^{3},\left|\Delta m^{3}\right|<\epsilon_{3}, \operatorname{sgn}\left(O_{A, B}\right)\right)-f_{4}\left(\Delta m^{4},\left|\Delta m^{4}\right|<\epsilon_{4}, \operatorname{sgn}\left(O_{A, B}\right)\right)
\end{array}
$$

where $f_{1}$ is an increasing function of $\Delta m^{1}$, while $f_{2}, f_{3}$ and $f_{4}$ are (non-linear) increasing functions of their gaps, but they also depend on the absolute value of the gap (if the gap is below a threshold, it is possible to have the opposite effect $)^{7}$, and on the signs of the possible outcomes of the two options (if among the outcomes there are losses, there could again be a contrasting effect). Moreover, we stop at the fourth moment given that results on the higher order, found in the cited literature (for instance, Deck and Schlesinger, 2014), show weak tendencies sometimes not statistically different from random choices.

\section{Financial risk aversion determinants}

In this section, we try to understand the possible determinants of financial risk aversion. To perform this task, we conduct an ordered logit analysis of the second, third, fourth moment and total "financial all moments risk aversion" scores on the following variables: age, gender, marital status, home (owned, rented...), labor status (employee, self-employed, unemployed...), social class (lower, lower-middle...), family income range (less than $5000 \$$, between $5000 \$$ and $20000 \$ \ldots$ ), number of vacations in the last year (as a proxy for the consumption propensity or as an alternative proxy for the level of income), level of education (primary, lower secondary...), and Country (we build a dummy only for areas with at least 10 observations, that is USA, India, Italy, Canada and Europe other than Italy; however, in the sample there are persons coming from Africa, Asia other than India, Latina America, and Australia). For a detailed description of the questions and of the possible choices, see Appendix A.

We perform various specifications of the ordered logit model with different explanatory variables, also using the stepwise selection procedure ${ }^{8}$. We will not report the tables with the coefficients, but we will summarize the main and most robust results ${ }^{9}$.

\footnotetext{
${ }^{7}$ Given that $f_{2}, f_{3}$ and $f_{4}$ are non-linear functions, we could omit the absolute value below a threshold assuming it is implied in the non-linear functions. However, we decide to insert it in order to remark the importance of this feature.

${ }^{8}$ The stepwise selection procedures consists of iteratively removing the explanatory variable with the highest pvalue above a certain threshold (we fix 10\%) and adding the variable with the lowest p-value below a certain threshold (we fix 5\%).

${ }^{9}$ All regressions are available from the authors upon request.
} 
- People prefer lower second moments (are risk averse) when they are older and/or they live in Europe, while they tend to be more risk prone (even if this result is not present in all the estimates done with different sets of explanatory variables) when they are employees and/or they live in India. The first result (on age) is in line with the assumptions usually done in financial asset management and confirms the findings of ?, but contrasts the findings of Noussair et al. [2014]. The result on geographical location, even if involving different countries, could present some similarities with the findings of other papers, that is people from "Western" countries seem to be more risk averse than people from "Eastern" countries; indeed ? report that Chinese respondents tend ( $10 \%$ p-value) to be less risk averse (second moment) and more edgy (fifth moment) than USA and German ones, and they report some references with the same results (for instance, ?, ?, find that Chinese people are more likely to take risks than Americans with respect to hypothetical payoffs). The weaker, but present, result on employee could be also matched with ? who find, at the weak p-value level of $10 \%$, that unemployed persons tend to be more risk averse in financial matters.

- Respondents prefer higher third moments (are prudent) if they are older and/or women. Moreover, Italians seem to be more prudent; however, this dummy is almost overlapping with the dummy "student" (that is statistically significant if we delete the country dummies), therefore we do not know if this result is driven by the fact that the Italians in the sample are almost all students of economics. The last result could be in line with the findings of Noussair et al. [2014] who state: "Students in the laboratory are more prudent than others, and higher education is correlated with greater prudence". The result on age is at odds with Noussair et al. [2014], who find a reduction in prudence with age, while the result on gender confirms the findings of Ebert and Wiesen [2014], even if they find that women are not only more "prudent", but also more "risk-averse" and "temperate" than men.

- Subjects prefer lower fourth moments (temperance) if they live in Europe (both Italy and Europe dummies are significant). Moreover, even if these results are not present in all the estimates, they tend to prefer lower fourth moments if they live in the USA, and higher fourth moments when they are single or employees.

The total score, composed by the three scores and mainly related to the second moment one, reflects the previous results. In particular, people present a higher risk aversion if they are older or live in "Western countries" (dummies Europe, Italy, Canada and USA).

All in all, some determinants (such as age) influence different moments, while other determinants seem to be specific of the risk aversion of a particular moment.

\section{Conclusions}

In this paper we face the very important economic topic of choices under uncertainty. Based on the theoretical evidence that higher moments of the return distribution matter in such decisions, we investigate how subjects take into account the first four moments of the return distribution. We propose a series of questions in which we manipulate the probability of winning as well as the outcomes in order to observe how subjects change their choices. We also modify the expected value of the lotteries in order to observe if the same choice is faced differently when the agents are in the "domain" of gains or losses. Moreover, we try to find the behavioral patterns of "all moments risk aversion" and "alternate propensity with even moments risk love", usually called "mixed risk aversion" and "mixed risk love".

We contribute to this literature because we perform a shorter and simpler questionnaire, and because 
we collect results from a very heterogeneous population (different countries and heterogeneous sociodemographic characteristics) by using the powerful tool of Amazon Mechanical Turk. Moreover, we build a "Rationality" score to see if there is substantial change in the results if we delete the less "rational" persons from the sample.

By using the data collected on the answers to the questionnaire, we confirm the well established result of the preference of the majority of the respondents for higher odd and lower even moments of the expected return distribution. However, we highlight many other features among which the three most relevant are:

- the importance of the gap between the values of the corresponding moments of the two choices. A large gap on a higher order moment can even drive the decision against the opposite preference on a lower order moment (we call this feature: " $n-t h$ moment very robust risk aversion"). In particular, the third (odd) moment seems more relevant even than the second (even) moment, confirming some literature findings, and explaining why risk averse persons participate to new year's lotteries or similar gambles. However, there should be a significant gap between the third moments of the two options, and results are less clear when both alternative are uncertain (high standard deviations) probably also because choices are more complicated (see the last item);

- the behavioral change in presence of a positive/zero/negative expected value. Indeed, jointly with the previous item, we can affirm that people are usually risk averse when the amounts are high or positive, but they are not risk averse when amounts are low and the expected value is zero or negative;

- the huge heterogeneity in behaviors. This could be due to the preference for a random choice when the choice overcomes a threshold of complexity (peculiar of every agent). Indeed, the complexity of the choice seems to be the most important driver of the propensity to switch from choosing on the basis of preferences to choosing randomly.

Finally, we analyse some possible determinants of risk propensity. We find that age and geographical location are very important determinants of risk propensity. Moreover, there are some other determinants that seem to influence the risk aversion related to a particular moment only. 


\section{References}

Maurice Allais. L'extension des théories de l'équilibre économique général et du rendement social au cas du risque. Econometrica, Journal of the Econometric Society, pages 269-290, 1953.

F. Arditti. Risk and the required return on equity. Journal of Finance, 22:19-36, 1967.

Thomas Astebro, José Mata, Luís Santos-Pinto, et al. Preference for skew in lotteries: Evidence from the laboratory. Paris, France: HEC Paris, 2009.

Thomas Astebro, José Mata, and Luís Santos-Pinto. Skewness seeking: risk loving, optimism or overweighting of small probabilities? Theory and Decision, 78(2):189-208, 2015.

Aurélien Baillon, Harris Schlesinger, and Gijs van de Kuilen. Measuring higher order ambiguity preferences. Experimental economics, 21(2):233-256, 2018.

M. Bar-Hillel. On the subjective probability of compound events. Organizational Behavior and Human Performance, 9(3):396-406, 1973.

M. Bernasconi. Nonlinear preferences and two-stage lotteries: Theories and evidence. Economic Journal, 104(1):54-70, 1994.

H. Bleichrodt and P. van Bruggen. Higher order risk preferences for gains and losses. Working paper, 2018.

Michael Buhrmester, Tracy Kwang, and Samuel D Gosling. Amazon's mechanical turk: A new source of inexpensive, yet high-quality, data? Perspectives on psychological science, 6(1):3-5, 2011.

H. Chiu. Skewness preference, risk taking and expected utility maximization. Geneva Risk and Insurance Review, 35:108-129, 2010.

D. Crainich, L. Eeckhoudt, and A. Trannoy. Even (mixed) risk lovers are prudent. American Economic Review, 103(4):1529-1535, 2013.

W. De Bondt, G. Muradoglu, H. Shefrin, and S.K. Staikouras. Behavioral finance: Quo vadis? Journal of Applied Finance, 18(2):7-21, 2008.

Cary Deck and Harris Schlesinger. Exploring higher order risk effects. The Review of Economic Studies, 77(4):1403-1420, 2010.

Cary Deck and Harris Schlesinger. Consistency of higher order risk preferences. Econometrica, 82 (5):1913-1943, 2014.

Cary Deck and Harris Schlesinger. On the robustness of higher order risk preferences. Working paper, 2016.

S. Ebert. Even (mixed) risk lovers are prudent: Comment. American Economic Review, 103(4): 1536-1537, 2013a.

S. Ebert. Moment characterization of higher-order risk preferences. Theory and Decision, 74: 267-284, 2013b.

Sebastian Ebert. On skewed risks in economic models and experiments. Journal of Economic Behavior \& Organization, 112:85-97, 2015. 
Sebastian Ebert and Daniel Wiesen. Testing for prudence and skewness seeking. Management Science, 57(7):1334-1349, 2011.

Sebastian Ebert and Daniel Wiesen. Joint measurement of risk aversion, prudence, and temperance. Journal of Risk and Uncertainty, 48(3):231-252, 2014.

Louis Eeckhoudt and Harris Schlesinger. Putting risk in its proper place. American Economic Review, 96(1):280-289, 2006.

Steinar Ekern. Increasing nth degree risk. Economics Letters, 6(4):329-333, 1980.

Z.G. Friedman. Testing the reduction of compound lotteries axiom: Violations in decision theory. Working Paper, Washington University, St. Louis, 2005.

Itzhak Gilboa and David Schmeidler. Maxmin expected utility with non-unique prior. Journal of Mathematical Economics, 18(2):141-153, 1989.

Uri Gneezy and Jan Potters. An experiment on risk taking and evaluation periods. The Quarterly Journal of Economics, 112(2):631-645, 1997.

Christian Gollier, James K Hammitt, and Nicolas Treich. Risk and choice: A research saga. Journal of risk and uncertainty, 47(2):129-145, 2013.

Leonard Green, Joel Myerson, and Pawel Ostaszewski. Amount of reward has opposite effects on the discounting of delayed and probabilistic outcomes. Journal of Experimental Psychology: Learning, Memory, and Cognition, 25(2):418, 1999.

Martin Halek and Joseph G Eisenhauer. Demography of risk aversion. Journal of Risk and Insurance, pages 1-24, 2001.

G.W. Harrison, J. MartÃnez-Correa, and J.T. Swarthout. Reduction of compound lotteries with objective probabilities: Theory and evidence. working paper, 2015.

C.R. Harvey and A. Siddique. Conditional skewness in asset pricing tests. Journal of Finance, 55 (3):1263-1295, 2000.

Timo Heinrich and Thomas Mayrhofer. Higher-order risk preferences in social settings. Experimental economics, 21(2):434-456, 2018.

John D Hey and Chris Orme. Investigating generalizations of expected utility theory using experimental data. Econometrica: Journal of the Econometric Society, pages 1291-1326, 1994.

Charles A Holt and Susan K Laury. Risk aversion and incentive effects. American economic review, $92(5): 1644-1655,2002$.

Philip A Horvath and Amit K Sinha. Asymmetric reaction is rational behavior. Journal of Economics and Finance, 41(1):160-179, 2017.

S. Huck and G. Weizacker. Risk, complexity, and deviations from expected-value maximization: Results of a lottery choice experiment. Journal of Economic Psychology, 20, 699-715, 1999.

E. Jondeau and M. Rockinger. Conditional volatility, skewness, and kurtosis: existence, persistence and comovements. Journal of Economic Dynamics and Control, Elsevier, 27:1699-1737, 2003.

Daniel Kahneman and Amos Tversky. Subjective probability: A judgment of representativeness. Cognitive Psychology, 1972. 
Alex Kane. Skewness preference and portfolio choice. Journal of Financial and Quantitative Analysis, 17(1):15-25, 1982.

Miles S Kimball. Precautionary saving in the small and in the large. Econometrica: Journal of the Econometric Society, pages 53-73, 1990.

Stanley J Kon. Models of stock returns -a comparison. The Journal of Finance, 39(1):147-165, 1984.

Alan Kraus and Robert H Litzenberger. Skewness preference and the valuation of risk assets. The Journal of Finance, 31(4):1085-1100, 1976.

Miriam Krieger and Thomas Mayrhofer. Prudence and prevention: An economic laboratory experiment. Applied Economics Letters, 24(1):19-24, 2017.

F. Lajeri-Chaherli. Proper prudence, standard prudence and precautionary vulnerability. Economics Letters, 82:29-34, 2004.

Graham Loomes and Robert Sugden. Regret theory: An alternative theory of rational choice under uncertainty. The economic journal, 92(368):805-824, 1982.

J. Maier and M. RÃ $\frac{1}{4}$ ger. Experimental evidence on higher-order risk preferences with real monetary losses. University of Munich Working Paper, 2012.

Harry Markowitz. Portfolio selection. The journal of finance, 7(1):77-91, 1952.

Charles N Noussair, Stefan T Trautmann, and Gijs Van de Kuilen. Higher order risk attitudes, demographics, and financial decisions. Review of Economic Studies, 81(1):325-355, 2014.

Amado Peiro. Skewness in financial returns. Journal of Banking \& Finance, 23(6):847-862, 1999.

John W Pratt. Risk aversion in the small and in the large. Econometrica, 32(1/2):122-136, 1964.

Drazen Prelec and George Loewenstein. Decision making over time and under uncertainty: A common approach. Management science, 37(7):770-786, 1991.

John Quiggin. A theory of anticipated utility. Journal of Economic Behavior \& Organization, 3 (4):323-343, 1982.

John Quiggin. Comparative statics for rank-dependent expected utility theory. Journal of Risk and Uncertainty, 4(4):339-350, 1991.

Paul A Samuelson. The fundamental approximation theorem of portfolio analysis in terms of means, variances and higher moments. The Review of Economic Studies, 37(4):537-542, 1970.

Robert C Scott and Philip A Horvath. On the direction of preference for moments of higher order than the variance. The Journal of Finance, 35(4):915-919, 1980.

Richard H Thaler, Amos Tversky, Daniel Kahneman, and Alan Schwartz. The effect of myopia and loss aversion on risk taking: An experimental test. The Quarterly Journal of Economics, 112(2): 647-661, 1997.

Stefan T Trautmann and Gijs van de Kuilen. Higher order risk attitudes: A review of experimental evidence. European Economic Review, 103:108-124, 2018.

T.C. Tsiang. The rationale of the mean-standard deviation analysis, skewness preference, and the demand for money. The American Economic Review, 62(3):354-371, 1972. 
Amos Tversky and Daniel Kahneman. Prospect theory: An analysis of decision under risk. Econometrica, 1979.

Amos Tversky and Daniel Kahneman. The framing of decisions and the psychology of choice. science, 211(4481):453-458, 1981.

Amos Tversky and Daniel Kahneman. Advances in prospect theory: Cumulative representation of uncertainty. Journal of Risk and uncertainty, 5(4):297-323, 1992.

H. von Gaudecker, A. Van Soest, and E. Wengstrom. Heterogeneity in risky choice behaviour in a broad population. American Economic Review, 101:664-694, 2011.

Bethany J Weber and Gretchen B Chapman. Playing for peanuts: Why is risk seeking more common for low-stakes gambles? Organizational Behavior and Human Decision Processes, 97(1):31-46, 2005. 


\section{Appendices}

\section{A Questionnaire}

0 You have the possibility to participate in a lottery in which you have $50 \%$ probability of winning $100 \$$ and $50 \%$ probability of winning nothing. There is no participation fee. By choosing Option A you participate in the lottery, otherwise by selecting Option B you do not participate. Which option do you choose? [A or B]

1 You have the opportunity to choose between two hypothetical investments: Option A offers the possibility to gain either $5 \$$ or $15 \$$ with $50 \%$ probability, Option B offers you a sure gain of $10 \$$. Which option do you choose? [A or B]

2 Suppose you have been fined and you have the opportunity to choose between two alternatives. Option A: pay a fine of $10 \$$. Option B: have a $50 \%$ probability of paying either $5 \$$ or $15 \$$. Which option do you choose? [A or B]

3 You may decide to participate to a lottery in which you have the possibility to gain or lose $5,000 \$$ with the same probability of $50 \%$. By choosing Option A you will take part in the lottery, otherwise by selecting Option B you do not participate. Which option do you choose? [A or B]

4 You may decide to take part in a lottery in which you have the possibility to gain or lose $5 \$$ with the same probability of $50 \%$. By choosing Option A you will take part in the lottery, otherwise by selecting Option B you do not participate. Which option do you choose? [A or B]

5 You have the possibility to choose between two hypothetical options. Option A: you have a $50 \%$ probability of winning or losing $5,000 \$$. Option B: you have a $50 \%$ probability of winning or losing $5 \$$. Which option do you choose? [A or B]

6 You have the possibility to choose between two hypothetical options. Option A: you have a $50 \%$ probability of winning either $5,000 \$$ or $15,000 \$$, Option B offers you a sure gain of $10,000 \$$. Which option do you choose? [A or B]

7 Suppose you have been fined and you have the opportunity to choose between two alternatives. Option A: pay a fine of $10,000 \$$; option B: have a $50 \%$ probability of paying either $5,000 \$$ or $15,000 \$$. Which option do you choose? [A or B]

8 You have the possibility to choose between two hypothetical options. Option A: you have a chance in a million to win a million Dollars and 0 otherwise. Option B: gain $1 \$$ for sure. Which option do you choose? [A or B]

9 You have the possibility to choose between two hypothetical options. Option A: lose 1,000\$ with a chance in a thousand and 0 otherwise. Option B: pay $1 \$$ for sure. Which option do you choose? $[\mathrm{A} \text { or } \mathrm{B}]^{10}$

10 You have the possibility to choose between two hypothetical options. Option A: 1\% probability of winning $10,000 \$$ and $99 \%$ probability of losing $101 \$$. Option B: $50 \%$ probability of winning $1,000 \$$ and $50 \%$ of losing $1,000 \$$. Which option do you choose? [A or B]

\footnotetext{
${ }^{10}$ We decide not to set the same absolute values of Question 8, in which we fix a winning amount equal to a million Dollars, in order to avoid a possible distorting effect: a person who does not have a million Dollars could decide to risk because in any case he/she will not pay the due amount.
} 
11 You have the possibility to choose between two hypothetical options. Option A: $1 \%$ chance to lose $10,000 \$$ and $99 \%$ chance to win $101 \$$. Option B: $50 \%$ probability to win $1,000 \$$ and $50 \%$ to lose $1,000 \$$. Which option do you choose? [A or B]

12 You are asked to choose between two hypothetical options with multiple outcomes. Option A: there is the possibility to gain, with the same chance equal to $33 \%, 0 \$$ or $5,000 \$$ or $10,000 \$$. Option B: there is the possibility to gain, with the same probability of $25 \%, 0 \$$ or $2,100 \$$ or $7,900 \$$ or $10,000 \$$. Which option do you choose? [A or B]

13 You are asked to choose between two hypothetical options with multiple outcomes. Option A: there is the possibility to get, with the same chance equal to $33 \%, 0 \$$ or $5,000 \$$ or $-5,000 \$$. Option B: there is the possibility to get, with the same probability of $25 \%, 2,900 \$$ or $5,000 \$$ or $-2,900 \$$ or $-5,000 \$$. Which option do you choose? [A or B]

Socio-demographic questions

- How do you see yourself: are you in general a person who takes risk or do you try to evade risk? Please self-grade your choice between 0 (I don't take risk at all) to 10 (I love to take risk).

- What is your age?

- What is your gender?

- In which country do you live now?

- What is your marital status?

o SINGLE;

o MARRIED;

o SEPARATED OR DIVORCED;

o WIDOW;

o OTHER.

- Describe the home where you live:

o IT IS OWNED BY YOU;

o IT IS RENTED FOR MONEY;

o IT IS OCCUPIED WITHOUT PAYMENT;

o I LIVE WITH FRIENDS;

o I HAVE NO PERMANENT RESIDENCE.

- How would you define your current labor status?

o EMPLOYEE;

o SELF-EMPLOYED;

o UNEMPLOYED AND LOOKING FOR A JOB;

o NOT WORKING AND NOT LOOKING FOR A JOB;

o STUDENT/UNPAID EXPERIENCE;

o RETIRED; 


\section{o FULFILLING DOMESTIC TASKS; \\ o OTHER.}

- Please choose one of the following that best describe your social class:
o LOWER;
o LOWER-MIDDLE;
o MIDDLE;
o UPPER-MIDDLE;
o UPPER.

- What is your total combined family income before taxes in the last 12 months from all sources?
o LESS THAN 5,000\$;
o $5,000 \$-19,999 \$$
o $20,000 \$-49,999 \$$
о $50,000 \$-149,999 \$$;
o MORE THAN 150,000\$;
o CHOOSE NOT TO ANSWER.

- What is the highest level of education you have completed?
o NO FORMAL EDUCATION;
o PRIMARY EDUCATION;
o LOWER SECONDARY EDUCATION;
o UPPER SECONDARY EDUCATION;
o POST-SECONDARY EDUCATION.

- In the last year, how many times did you go on vacation?
o NONE;
o ONCE;
o TWICE;
o MORE THAN TWICE.

- For each of the following statements, please indicate your likelihood of engaging in each activity or behavior. Provide a rating from 1 (Very unlikely) to 5 (Very likely).

- Cheating in an exam;

- evading tax;

- smoking;

- periodically engaging in a dangerous sport;

- exposing yourself to the sun without using sunscreen;

- going on a vacation in third-world country without prearranged travel and hotel accommodation;

- shoplifting a small item (e.g a pen or a lipstick);

- driving your car without fastening seat-belt;

- regularly eating high cholesterol foods.

- How old were you 10 years ago? [Control question] 


\section{B Terminological and methodological issues}

\section{B.1 Risk apportionment and complexity}

Scott and Horvath [1980] prove the importance of higher distributional moments when the EU theory holds. However, the experimental literature on higher order risk preferences develops much later from the milestone study of Eeckhoudt and Schlesinger [2006]. They implement a modelindependent way to study higher-order risk attitudes without the assumptions of the EU, and coin the terminology "risk apportionment of order $n$ ". Abstracting from a utility function (with its derivatives), in the recent behavioral finance literature, even the definitions of prudence and temperance slightly change. Indeed, prudence is defined as "downside risk aversion", and temperance as "outer risk aversion". Under differentiable utility functions, both risk aversion, prudence, and temperance definitions are equivalent. More generally, "risk apportionment of order $n$ " is equivalent to Ekern [1980] "n-th degree risk aversion".

The Eeckhoudt and Schlesinger [2006] study, as already mentioned, constitutes a reference point in the experimental literature to directly measure prudence, temperance and risk apportionment of higher orders. An example of the risk apportionment task is shown in Figures 2 and 3. The task consists in choosing among two multi-armed lotteries which usually exhibit the same first and second moments but vary in terms of either the third or the fourth. Figure 2 represents an example of a task to measure prudence. We assume that the outcomes $x, y$ and $+z_{1},-z_{1}$ are equally probable and that $y$ is smaller than $x$. Therefore, we add in one of the two outcomes a zero-mean risky option. The sole difference between the lotteries presented is that in lottery $\mathrm{L}$ the risky part is added to the highest value while in lottery $\mathrm{R}$ it affects the lower outcome. Choosing $\mathrm{L}$ over $\mathrm{R}$ reveals prudence. In other words, prudent people prefer to add a zero-mean risk to the better o utcome in a binary lottery or to "combine good with bad".

Temperance, instead, is described by Eeckhoudt and Schlesinger [2006] as preference for "disaggregating the harms", that is again avoiding to "combine bad with bad". Figure 3 shows that the only difference between the two lotteries lies in having a zero-mean risky option spread over the two options (lottery L) or having a cumulative risk in one of the two options (lottery R). Notice that, in this case, there is no difference in the expected value of the outcome $x$. A subject who chooses lottery L, that is a subject who prefers to spread the two zero-mean risks, is said to be temperate. This mechanism can be replicated for higher order risk apportionment, and people who present "risk apportionment" are always defined as agents who prefer to "combine good with bad", that is they prefer to risk when they are richer and to disentangle risks.

If "n-th degree risk aversion" is restricted to the field of EU theory, also the risk apportionment task and the related definitions of prudence, temperance and risk apportionment of order $\mathrm{n}$, restrict the analysis to some peculiar mechanisms that we think can be generalized within the framework of

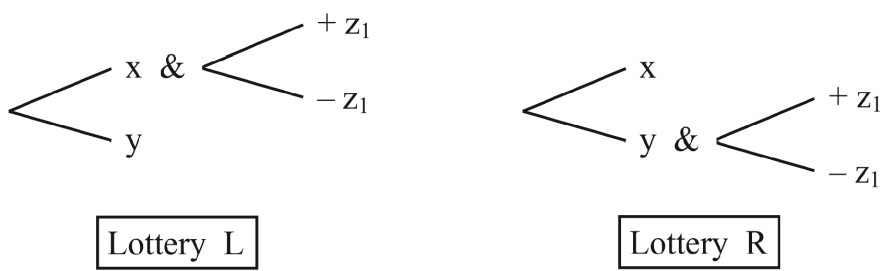

Figure 2: Example of binary choice task identifying prudence. $x, y$, and $z_{1}$ are positive numbers with $x>y$. This is the same example proposed in Noussair et al. [2014] 


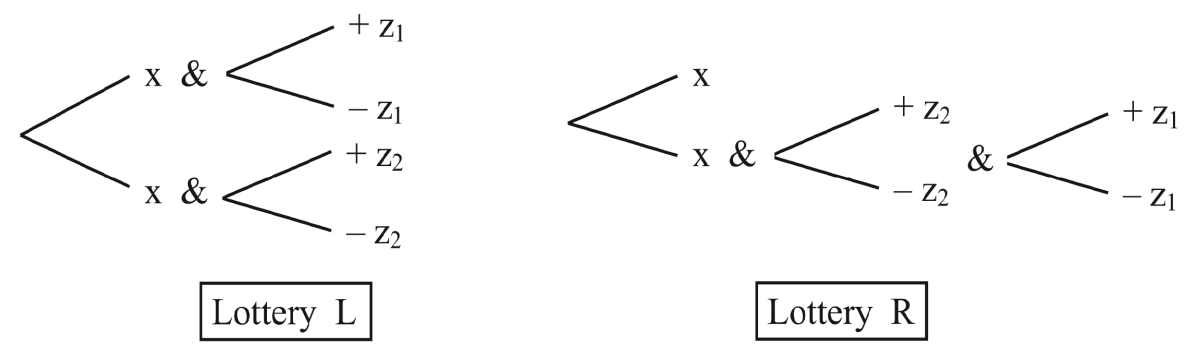

Figure 3: Example of binary choice task identifying temperance. $x, z_{1}$, and $z_{2}$ are positive numbers. This is the same example proposed in Noussair et al. [2014]

"n-th moment risk aversion"11. First, the prudence definition of "downside risk aversion" implies a behavior aimed at avoiding a choice presenting a very large negative third moment, but it does not imply preferring a high positive to a low positive third moment value. In this paper, we have shown that people have a preference for a higher value of the third moment, even if it can not be seen as a "prudent" choice (see Question 7 of our questionnaire) and, therefore, beyond the restrictive feature of prudence.

Secondly, the risk apportionment task is restrictive and relatively complex, because the restriction of the analysis to symmetric 50\%-50\% zero mean risks creates outputs with an unnecessary high number of possible outcomes. For instance, to analyse prudence, lotteries L and R reported in Figure 2 present both three outcomes, while we can study the more general feature of "third moment risk aversion" comparing lotteries with two outcomes (see Questions 9 and 10 of our questionnaire). This feature holds for higher order risk apportionment: for instance, Figure 3 shows a lottery (L) with 4 outcomes and a lottery $(\mathrm{R})$ with 5 outcomes, while we could study the "fourth moment risk aversion" using simpler lotteries with 3 outcomes only.

In our questionnaire, we try to be as simple as possible, because when the question is too complex, a lot of agents tend to reply randomly. Noussair et al. [2014] explain that the complexity of the tasks is especially important when non-student subjects are employed, because they could have very heterogeneous educational levels. Deck and Schlesinger [2014] attribute the weak significance of the results on fifth and sixth order attitudes "to the ever increasing complexity involved with deciphering higher degrees of risk increases".

The complexity issue is related to the issue about the choice between compound and reduced lotteries. Deck and Schlesinger [2014] explain that almost all experiments on the risk apportionment task use compound lottery in order to make evident the interpretation of the problem as "combining good with good" versus "combining good with bad". Instead, we use reduced form lotteries because we do not focus on the previous interpretation, but we care about the simplicity of the questionnaire. Deck and Schlesinger [2014] report that "Huck and WeizÃơcker [1999] examine deviations from maximizing the expected payoff and find that subjects care less about $2 n d$ order risk when the tasks become more complex. This idea seems to also hold for higher orders". They add that the "paper by Maier and RÃ $\frac{1}{4}$ ger [2012] does just the opposite and present lotteries for risk attitudes of orders 2-4 only in a reduced form. Their basic results do not differ from the other experiments to date, each of which presents the choices as compound lotteries" and that "we do not see any strong evidence against reduction of compound lotteries". Deck and Schlesinger [2016] and ? perform a direct comparison between answers to the same questions proposed in a compound and reduced frame, even if both experiments are based on quite a small sample (53

\footnotetext{
${ }^{11}$ Ebert [2013b] explains the relationship between moments of the return distribution in these lotteries and prudence and temperance.
} 
and between 70 and 73 participants for different questions, respectively). On one hand, Deck and Schlesinger [2016] find that people's behavior under one format has little bearing on their choice under the other format, with low correlation for prudence and temperance and no correlation for edginess; moreover, they find that in the aggregate people are more temperate and less edgy with compound lotteries, while? find that prudence and temperance are stronger for compound lotteries than for reduced ones, and that there is no impact on edginess and sixth order risk apportionment. We think that these results can be a clue of the presence of a large number of random choices due to the fact that questions are complex, with a lot of possible outcomes which are sometimes not very different among themselves. On the other hand, both Deck and Schlesinger [2016] and ? find that aggregate patterns are not much different; for instance, ? report that people prefer the less risk loving alternative for order 3 to 5 in both formats, and that choices for order 6 are not statistically different from random behavior in both formats, with the only difference that the distribution of choices for order 4 is not statistically different (10\% p-value) from the distribution expected under random behavior only for redu ced lotteries. The comparison of the results obtained by compound and reduced lotteries is beyond the purposes of this paper. However, we think that aggregate results should not radically change between the two formats. Moreover, between the two kinds, as already explained, we prefer the simpler reduced form. Indeed, the cited Maier and R $\tilde{A} \frac{1}{4}$ ger [2012] (even if they require a very long task of 84 binary choices) explain that "we rather presented the gambles in final outcomes. We chose this design, as it is well established in the literature that in experiments subjects have severe difficulties in solving compound lotteries (see e.g. Bar-Hillel, 1973; Tversky and Kahneman, 1979, in their discussion of the isolation effect; Bernasconi, 1994; or Friedman, 2005)." In addition, the relatively complex compound method can present another specific framing problem highlighted by Ebert and Wiesen [2011]: are individuals' decisions influenced by the fact that they are asked to add the zero-mean risk or the fixed amount to a state of the 50/50 gamble? Moreover, Harrison et al. [2015], citing ?, explain that there could be the "possibility of having a distinct (dis)utility for the act of gambling on compound lotteries"; this feature can distort the results obtained by compound lotteries if the payment procedure is based on the drawing of one random question which is really paid.

\section{B.2 Terminological issues}

Going back to the terminological issues, if prudence is not the same as "third moment risk aversion", it is also different from "skewness seeking", as explained by Ebert and Wiesen [2011]. Indeed, they explain that prudence implies preferring the choice with the better skewness even if this choice presents a worse kurtosis. Therefore, two consequent relevant definitions are the following (Ebert, 2013b, and Ebert and Wiesen, 2011):

- the "kurtosis robustness feature of prudence" (or "kurtosis robustness feature of higher-order risk preferences of odd order") implies that a lottery with the higher skewness is preferred whatever the values of kurtosis and of the other higher even moments;

- the "skewness robustness feature of temperance" (or "skewness robustness feature of higherorder risk preferences of even order") implies that a lottery with the lower kurtosis is preferred whatever the values of the odd moments of order higher than 4 .

In reality, these two definitions can beget confusion and are usually not feasible. Indeed, as shown by our experiment, the gap between moments is relevant. Indeed, for instance, we can assume that the agent, as usual, prefers a lower value of the second moment and a higher value of the third one, and that option A presents a lower value of both the second and the third moments, while option B 
presents higher values. It could happen that the agent chooses option A when the gap on the second moment is relevant and the gap on the third moment is low, while chooses option B when the gap on the third moment is high and the gap on the second moment is low. This is in line with the vast and well established literature on certain equivalent and utility premium: a risk averse person can choose the safer option if the positive gap on the second moment is relatively large compared to the positive gap on the first moment, and the same person can choose the riskier option if the positive gap on the second moment is relatively small compared to the positive gap on the first moment (there is enough risk premium). This feature is very evident in parado xical situations such as the one proposed in our Question "zero" (see Section 3.3), where the worst outcome of the risky option is as good as the certain outcome of the safe option.

In order to avoid confusion, we suggest the following definitions that we will use in the paper:

- an agent presents the " $n$-th moment risk aversion" if she prefers: (i) a lower value of the analysed even moment, all other moments being equal, or (ii) a higher value of the analysed odd moments, all other moments being equal ${ }^{12}$;

- an agent presents the " $n$-th moment robust risk aversion" if she prefers, at least for some ranges of the values: (i) a lower value of the analysed even moment, all the lower moments being equal and regardless of the value of the higher moments (this is similar to "skewness robustness feature of temperance"), (ii) a higher value of the analysed odd moment, all the lower moments being equal and regardless of the value of the higher moments (similarly to "kurtosis robustness feature of prudence");

- an agent presents the " $n$-th moment very robust risk aversion" if she prefers, at least for some ranges of the values: (i) a lower value of the analysed even moment regardless of the value of the other moments, (ii) a higher value of the analysed odd moment regardless of the value of the other moments.

The "n-th moment risk aversion" is quite easily testable in an experiment, while the other two features, that are coupled, are more complex, because they could be found for the same agent only for some values of the moments. For sure if the $n-t h$ moment presents "robust risk aversion", the moments of order higher than $n$ can not present "very robust risk aversion" and vice versa, therefore the two features (if one is present) are mutually exclusive, at least for a given range of the values of the moments.

Other definitions try to describe the whole behavior of the agent:

- "risk aversion", and "mixed risk aversion" (?, ?) have the same meaning, that is the derivatives of the utility function alternates their sign with the first derivative strictly positive, as in Scott and Horvath [1980];

- "risk lovers" present the opposite pattern to a "risk averse" person;

- "mixed risk lovers" have all derivatives of the utility function strictly positive. Therefore, as explained by Crainich et al. [2013], they are prudent even if they are risk lovers on variance.

We suggest to read Ebert [2013a] for a clear explanation of the implications of these terms. Again, these definitions can cause confusion in discussions, therefore we will use them in the paper with the following modifications:

\footnotetext{
${ }^{12}$ In the EU theory, this result is equivalent to the theoretical prediction of Scott and Horvath [1980] and Ekern [1980] and, related to the third moment but outside the EU theory, it is equivalent to the "skewness seeking" definition of Ebert and Wiesen [2011].
} 
- "all moments risk aversion" for a person with a preference for higher odd moments and lower even moments (in the EU theory, "risk averter", and "mixed risk averter");

- "all moments risk love" for a person with a preference for lower odd moments and higher even moments ("risk lovers");

- "alternate propensity with even moments risk love" for a person who prefers higher values for all moments ("mixed risk lovers").

In this way, the definitions are not ambiguous. Moreover, we could potentially create other cases, even if the literature points out that "mixed risk averter" and "mixed risk lovers" are the most common kind of agents found.

\section{Results on second moment analyses}

\section{C.1 Small second moment gap}

We start with questions 1,2 and 4. Each question presents two options with the same expected value, but a different second moment: an option with a small variance and an even safer option with zero variance, that is a sure choice. The three questions have the same structure in terms of variance, but they differ for the expected value: Question 1 has a small gain (small positive expected value) equal to $10 \$$, Question 2 presents a small loss (small negative expected value) equal to $-10 \$$ and Question 4 has zero expected value.

As already explained in Section 4.1, when the expected value is positive, people usually prefer the sure gain (almost 70\% of respondents chooses answer B in Question 1), that is there is risk aversion. Instead, when the expected value is negative, preferences between a sure loss or a variable loss are equally divided, indeed the answers in Question 2 are not statistically different from a $50 \%-50 \%$ division. Lastly, when the expected value is zero, people often decide to participate in the lottery $(59 \%)$ showing a risk prone behavior.

Table 7 deepens the analysis showing the joint behavior of the respondents to the three questions. Observing Table 7, we can state that:

- the largest group $(20.08 \%)$ is risk averse over gains and risk prone when the expected value is zero or negative (answers B-B-A respectively to questions 1-2-4), showing one risk preference inversion.

- The second largest group is composed of the consistently risk averse people (B-A-B answers) and regards $18.83 \%$ of respondents. Instead, $11.92 \%$ are consistently risk prone (A-B-A), therefore $30.75 \%$ of our subjects are consistent in their risk propensity.

- The third largest group includes $18.20 \%$ of subjects who answer B-A-A. These people tend to be risk averse when the expected amount is different from zero and risk prone when the expected amount is zero. This pattern presents two risk preference inversions, that could be caused by the so-called "peanuts effect": when the expected value is zero people like to bet, while they avoid risk when the expected value is far from zero. However, also in questions 1 and 2 the variance of the return is very small and the expected value is not very far from zero, therefore we classify these people as "inconsistent" including $3.97 \%$ of people who answer A-B-B, for a total of $22.18 \%$ of "inconsistent" subjects who show two inversions in risk preference. 
Table 7: Joint distribution of replies to Questions 1-2-4.

\begin{tabular}{|c|c|c|c|c|}
\hline & q2-A/q4-A & q2-B/q4-A & q2-A/q4-B & q2-B/q4-B \\
\hline q1-A & 9.00 & 11.92 & 5.44 & 3.97 \\
\hline q1-B & 18.20 & 20.08 & 18.83 & 12.55 \\
\hline
\end{tabular}

- The last group including more than $10 \%$ of respondents (that is $12.55 \%$ who always answer B) is the group of people that are risk averse when the expected value is positive or zero and risk prone in losses, showing one risk preference inversion.

All in all, when the gap in the second moment between the two options and the amounts are low, we show a huge heterogeneity in behaviors, with different responses to a second moment increase over gains, losses and when the expected value is zero.

\section{C.2 Large second moment gap}

Questions 3,6 and 7 present two options with the same expected value and a very different second moment. All questions have the same variance gap between the two options, but they present three different expected values: Question 3 has zero expected value, while Question 6 has a positive expected value, and Question 7 has a negative one.

As already explained in Section 4.1, the vast majority of people is risk averse when the lottery has a positive expected value: almost $80 \%$ of subjects answer B to Question 6, that is they prefer the sure gain.

People tend to be risk averse also when the lottery has zero or a negative expected value, but in this case there is a much larger percentage of subjects that choose the risky option: $40 \%$ and $45 \%$ or respondents respectively. However, the percentage of people who choose the safe option is above and statistically different from $50 \%$ even when the expected value is negative $(54.81 \%$ in Question $7)$.

Table 8 presents the joint behavior of the respondents to the three questions. More than half of the respondents are included in the following two groups:

- $28 \%$ of subjects are consistently risk averse, that is they always choose the safe option with zero second moment (q3-B, q6-B, q7-A);

- $24 \%$ of respondents are risk averse when the expected value is positive or zero and risk prone when the expected value is negative (q3-B, q6-B and q7-B).

However, about $48 \%$ of respondents show a strong heterogeneity in behaviors: for instance almost $15 \%$ prefer the safe option when the expected value is far from zero and choose the risky one when the expected value is zero, perhaps showing a tendency to avoid extreme outcomes, while $12 \%$ of subjects is risk averse over gains and risk prone when the expected value is zero or negative. We highlight that only $5.44 \%$ of persons are consistently risk prone, that is they always choose the option with higher second moment: this could be a strong clue that people are not risk prone when the gap between the second moment of the two options is high, and that some risky choices could be due to random choices.

We can strengthen the previous results, narrowing the analysis to the joint behavior of the respondents to two of the three mentioned questions, and in particular to Questions 6 and 7 as reported in Table 9 (however, we obtain similar results using Question 3 and 6). Indeed, we clearly find two major groups: almost $43 \%$ of respondents are consistently risk averse people (q6-B and 
Table 8: Joint distribution of replies to Questions 3-6-7.

\begin{tabular}{|c|c|c|c|c|}
\hline & q6-A/q7-A & q6-B/q7-A & q6-A/q7-B & q6-B/q7-B \\
\hline q3-A & $7,32 \%$ & $14,85 \%$ & $5,44 \%$ & $11,92 \%$ \\
\hline q3-B & $4,60 \%$ & $28,03 \%$ & $4,18 \%$ & $23,64 \%$ \\
\hline
\end{tabular}

Table 9: Joint distribution of replies to Questions 6-7.

\begin{tabular}{|l|c|c|c|}
\hline & q7-A & q7-B & Total \\
\hline q6-A & 11.92 & 9.62 & 21.55 \\
\hline q6-B & 42.89 & 35.56 & 78.45 \\
\hline Total & 54.81 & 45.19 & 100.00 \\
\hline
\end{tabular}

q7-A), and almost $36 \%$ of respondents are risk averse over gains and risk prone over losses (q6-B and $\mathrm{q} 7-\mathrm{B})$.

Summarizing, when the gap between the second moments of the two options is high, we can state that: (i) a lot of people are consistently risk averse irregardless of the expected value; (ii) there is quite a large group of subjects that show a different risk propensity when the expected value is positive or negative and, in particular, they are risk prone in losses; (iii) consistently risk prone subjects are almost nonexistent.

\section{C.3 Low vs high second moments gaps}

\section{C.3.1 Questions 3-4-5: zero expected value}

Questions 3, 4 and 5 present two options with same expected value equal to zero, but different second moments and, in particular, a sure option (safe choice, with zero variance) in Questions 3 and 4. Among the three questions, Question 4 presents a low gap between the variance of the two choices, while Questions 3 and 5 have a larger variance gap between the two options, that is the bet in Questions 3 and 5 present larger amounts. Between Questions 3 and 5 the difference is that Question 3 presents a sure choice, while both choices in Question 5 have a positive second moment.

As observed in Appendix C.1, the majority of people tend to be risk prone when the lottery is for a low amount (59\%), but Question 3 and 5 show that they become risk averse when the stake is large ( $60 \%$ and $66 \%$ respectively).

Table 10 presents the joint behavior of the respondents to the three questions. We can find three major groups:

- $26.15 \%$ of persons are consistently risk averse, that is they always choose the option with lower second moment (they always answer B);

- $23.85 \%$ of subjects are risk prone when the gap in the second moment value between the two choice is low and risk averse in the opposite case, or risk prone for low amounts and risk averse for large amounts (q3-B, q4-A and q5-B), consistent with a possible "peanuts effect";

- $19.04 \%$ of persons are consistently risk prone, that is they always choose the option with higher second moment (they always answer A).

These three groups cover almost $70 \%$ of the sample. However, a $31 \%$ of subjects cannot be easily classified and, in some cases, "rationally" understood. 
Table 10: Joint distribution of replies to Questions 3-4-5.

\begin{tabular}{|c|c|c|c|c|}
\hline & q4-A/q5-A & q4-B/q5-A & q4-A/q5-B & q4-B/q5-B \\
\hline q3-A & 19.04 & 4.81 & 10.04 & 5.65 \\
\hline q3-B & 6.28 & 4.18 & 23.85 & 26.15 \\
\hline
\end{tabular}

Table 11: Joint distribution of replies to Questions 3-4.

\begin{tabular}{|l|c|c|c|}
\hline & q4-A & q4-B & Total \\
\hline q3-A & 29.08 & 10.46 & 39.54 \\
\hline q3-B & 30.13 & 30.33 & 60.46 \\
\hline Total & 59.21 & 40.79 & 100.00 \\
\hline
\end{tabular}

As already explained in Section 3.3, Questions 3-4-5 are also implemented to compute the "Rationality" score. For instance, a subject choosing option A in Question 4 (preference for the small bet compared to the safe option) and option A in Question 5 (preference for the large bet compared to the small bet), for the transitivity of preferences, should choose option A in Question 3 too (preference for the large bet compared to the safe choice). Therefore, the sequence 3B-4A-5A violates the transitivity of preferences and can be defined "irrational". The same holds for the sequence 3A-4B-5B. These two groups sum up almost $12 \%$ of respondents.

Narrowing the analysis to the joint behavior of the respondents to two of the three mentioned questions, and in particular to Questions 3 and 4, we can observe in Table 11 that the population can be almost equally divided in three major groups:

- consistently risk averse persons (q3-B and q4-B: 30.33\%),

- consistently risk prone persons (q3-A and q4-A: 29.08\%),

- subjects who are risk prone for low amounts and risk averse for large amounts (q3-B and q4-A: 30.13\%), consistent with a possible "peanuts effect".

Risk prone people for a large second moment and risk averse for a low second moment are quite rare $(10 \%)$. These results confirm what we observed in Table 10, and a very similar result can be obtained observing the joint distribution to Questions 4 and 5.

Summing up, we show that the value (or the gap between the two options) of the second moment is an important determinant of the choice because, beside the two groups of risk averse and risk prone persons, there is another large group of subjects who are risk prone for a low second moment and risk averse for a large one.

\section{C.3.2 Questions 1-6 and 2-7: gain and loss}

In this subsection we compare both Questions 1-6 and 2-7 to check whether there are significant differences in subjects' behavior when the expected value is either positive or negative. As already explained in Section 4.1, Questions 1 and 6 present two options with positive expected value, but different second moments. The same holds for Question 2 and 7, but for the expected values that is negative in both questions.

The first result is that risk aversion increases when the second moment gap increases: a larger percentage of respondents chooses the safe choice comparing Question 6 with Question 1 (option B) and Question 7 with Question 2 (option A). Moreover, the results in Table 12 show that Questions 
Table 12: Joint distribution of replies to Questions 1-6 and 2-7.

\begin{tabular}{|l|c|c|c|}
\hline Panel A & q6-A & q6-B & Total \\
\hline q1-A & 13.18 & 17.15 & 30.33 \\
\hline q1-B & 8.37 & 61.30 & 69.67 \\
\hline Total & 21.55 & 78.45 & 100.00 \\
\hline \hline Panel B & q7-A & q7-B & Total \\
\hline q2-A & 33.26 & 18.20 & 51.46 \\
\hline q2-B & 21.55 & 26.99 & 48.54 \\
\hline Total & 54.81 & 45.19 & 100.00 \\
\hline
\end{tabular}

6 and 7, that is questions with large amounts and large second moment gaps, strengthen the results of Question 1 and 2, that is questions with low amounts and low second moment gaps.

Observing the joint distribution, starting from panel A (positive expected value), we see that:

- people are usually consistently risk averse over gains, indeed $61.30 \%$ of respondents always prefer option B (the sure gain);

- more than a fourth of respondents $(8.37 \%+17.15 \%)$ are "inconsistent" for different amounts / std. However, $17.15 \%$ of subjects are initially risk loving then risk averse over gains, and this pattern is consistent with the "peanuts effect", but also with the Markowitz theory as explained by Georgalos et al. (2018): "Markowitz assumed that (...) the subject was initially risk loving then risk averse over gains, whilst initially risk averse then risk seeking over losses". Instead, 8.37\% of subjects, who choose B for Question 1 and $\mathrm{A}$ for Question 6, seems to like to bet, but only if the stake is large; however this behavior is not confirmed by the choices in the other analysed questions, therefore we can assume it is an "irrational" or "random" behavior;

- only $13.18 \%$ of subjects are consistently risk prone over gains.

Panel B of Table 12 (negative expected value) shows that over losses people are very heterogeneous:

- $33 \%$ of respondents are consistently risk averse;

- $27 \%$ of subjects are consistently risk prone;

- $22 \%$ of people are risk loving for low second moment gaps/amounts and risk averse for large second moment gaps/amounts, confirming that risk aversion increases when the second moment increases;

- $18 \%$ of persons are risk averse for low second moment gaps/amounts and risk prone for large second moment gaps/amounts, as theorized by Markowitz. Therefore, our results reject that model (at least for the vast majority of people), even if, as predicted, there is a different behavior between gains and losses, and risk aversion increases over gains.

To wrap up, we can state that: (i) risk aversion increases when the second moment increases; (ii) subjects are usually risk averse in gain; (iii) there is high heterogeneity in behaviors when the expected value is negative. 


\section{Results on third moment analyses}

In this section, we focus on the behavior of our respondents to choices that involve a different third moment of the distribution of returns (and different other moments too, always keeping the same expected value between the two options).

\section{D.1 Positive odd (third) higher moments}

In this subsection, we analyse what happens in a choice with an option containing a pay-off with higher positive third moment, studying Questions 8 and 10 .

In Question 10 both options have the same first and second moments, but Option A presents larger higher order moments. Therefore, a risk averse (including "risk aversion", "prudence" and "temperance" in the risk aversion concept) person should prefer Option B because of its lower even moments, but should prefer Option A because of its higher odd moments. As already explained in Section 4.2, Option A has the "third moment robust risk aversion" feature, while Option B has the "fourth moment very robust risk aversion" feature. The results show that the sample is almost equally divided (not statistically different from a random choice), confirming the "struggle" between two opposite effects without a prevalence.

Questions 8 presents two options with the same expected value, but Option A presents larger higher order moments (including the second moment). A risk averse subject should prefer lower even moments (option B) and higher odd moments (option A). Option A has the "third moment very robust risk aversion" feature (third moment even more important than second one), while Option B has the "second moment robust risk aversion" feature.

The result is very strong: almost the $70 \%$ of respondents prefer Option A, that is they show the "third moment very robust risk aversion". The reason for the different behavior between Questions 8 and 10, could be the gap between the third moment of the two options, which is much larger in Question 8 compared to Question 10. In other words the third (odd) moment could be more relevant even than the second (even) moment, but the gap between the third moments of the two should exceed a very significant threshold.

As explained in Appendix B.2, the strong preference for option A is a very relevant result. In this question, we could hardly define Option A as the choice of a "prudent" person. It shows that the prudence feature, defined as "downside risk aversion", represents only a subset of behavior due to the more general preference for a higher third moment. Indeed, prudence is aimed at avoiding a large loss, that is a largely negative third moment, but it does not imply to prefer a high positive third moment compared to a low positive third moment value (unless we want to force the interpretation as a regret for the loss of a large gain). Instead, our result shows that people always tend to prefer a higher third moment.

We can add that the tendency to choose option A is stronger for people with a higher value of the "Rationality" score, that is people who could be more intelligent, or who could have compiled the questionnaire with more care: $71.83 \%$ of respondents who obtain a "Rationality" score of 4 or 5 choose option A, while only $61.94 \%$ of subjects who obtain less than 4 choose option A.

Table 13 reports the joint distribution of answers to Question 8 and 10. The largest group is the one that chooses Option A in both questions, therefore the majority of the people presents the "third moment very robust risk aversion". This table shows a tendency to be consistent between the two choices, even if it is weak: slightly more than half of the respondents who choose Option A in Question 8 tend to prefer Option A also in Question 10 (35.15\% vs 33.47\%), and slightly more than half of the respondents who choose Option B in Question 8 tend to prefer Option B also in Question 10 (16.95\% vs $14.44 \%)$. 
Table 13: Joint distribution of replies to Questions 8 and 10.

\begin{tabular}{|l|c|c|c|}
\hline & q10-A & q10-B & Total \\
\hline q8-A & 35.15 & 33.47 & 68.62 \\
\hline q8-B & 14.44 & 16.95 & 31.38 \\
\hline Total & 49.58 & 50.42 & 100.00 \\
\hline
\end{tabular}

Table 14: Replies to Questions 9 and 11 dividing respondents by second moment "financial risk aversion score".

\begin{tabular}{|l|c|c|c|c|c|c|c|c||c|}
\hline 2nd moment risk aversion & 0 & 1 & 2 & 3 & 4 & 5 & 6 & 7 & Total \\
\hline \hline q9-A & 1 & 8 & 21 & 34 & 32 & 24 & 11 & 4 & 135 \\
\hline q9-A\% & 33.33 & 42.11 & 41.18 & 43.04 & 25.40 & 25.53 & 18.33 & 8.70 & 28.24 \\
\hline q9-B & 2 & 11 & 30 & 45 & 94 & 70 & 49 & 42 & 343 \\
\hline q9-B\% & 66.67 & 57.89 & 58.82 & 56.96 & 74.60 & 74.47 & 81.67 & 91.30 & 71.76 \\
\hline Total & 3 & 19 & 51 & 79 & 126 & 94 & 60 & 46 & 478 \\
\hline \hline q11-A & 2 & 10 & 33 & 51 & 76 & 50 & 41 & 29 & 292 \\
\hline q11-A\% & 66.67 & 52.63 & 64.71 & 64.56 & 60.32 & 53.19 & 68.33 & 63.04 & 61.09 \\
\hline q11-B & 1 & 9 & 18 & 28 & 50 & 44 & 19 & 17 & 186 \\
\hline q11-A\% & 33.33 & 47.37 & 35.29 & 35.44 & 39.68 & 46.81 & 31.67 & 36.96 & 38.91 \\
\hline Total & 3 & 19 & 51 & 79 & 126 & 94 & 60 & 46 & 478 \\
\hline
\end{tabular}

To sum up, we find that the majority of the people presents the "third moment very robust risk aversion" when there is a strong gap in the value of the third moment, that is the third (odd) moment seems even more relevant than the second (even) moment (confirming literature findings about the importance of "prudence"), but there should be a significant gap. With a smaller third moment gap, almost half of the people that show the "third moment very robust risk aversion", do not present it anymore and they do not even show "third moment robust risk aversion".

\section{D.2 Negative odd (third) higher moments}

In this subsection, we analyse what happens in a choice with an option containing a pay-off with a highly negative third moment, centering the attention on Questions 9 and 11.

We start our analysis with Question 11: both options have the same first and second moments, but Option A presents larger negative higher order odd moments and larger positive higher order even moments. In this case, a risk averse person should surely prefer Option B because of its higher odd moments (odd moments are zero because Option B is symmetrical, while they are negative in Option A) and lower even moments of the pay-off. Surprisingly, persons usually prefer Option A (more than $61 \%$ of respondents).

One may guess that there is a correspondence between the risk aversion shown by respondents on the second moment and the behavior in Question 11, indeed risk prone people could prefer Option A. Table 14 panel B reports the choice dividing respondents with the value of risk aversion on second moment that they have obtained (see Section 3.4). The distribution shown in Table 14 does not support our guess since there is not a clear pattern.

Question 9, again, presents two choices with the same expected value but different higher order moments. Indeed, Option A presents larger negative higher order odd moments and larger positive higher order even moments (including the second moment). Following Scott and Horvath [1980], a 
Table 15: Joint distribution of replies to Questions 9 and 11.

\begin{tabular}{|l|c|c|c|}
\hline & q11-A & q11-B & Total \\
\hline q9-A & 18.41 & 9.83 & 28.24 \\
\hline q9-B & 42.68 & 29.08 & 71.76 \\
\hline Total & 61.09 & 38.91 & 100.00 \\
\hline
\end{tabular}

risk averse subject should surely prefer Option B (higher odd moments and lower even moments). Table 1 shows a strong result: people usually tend to be risk averse and "prudent", indeed $72 \%$ of respondents choose Option B. Moreover, Table 14 panel A reports the choice dividing the respondents with the value obtained on second moment risk aversion score. We can observe that there is a correspondence between the risk aversion shown by respondents on the second moment and the behavior in Question 9: when the risk aversion increases, the percentage of people who prefer the safer option (Option B) increases.

Two opposite results emerge from the analysis of the two questions. Table 15, that reports the joint distribution of answers to Question 9 and 11, strengthens this finding. Indeed, it shows that almost half of the sample (43\% of subjects) answer B to Question 9 and A to Question 11. A possible explanation of the choice of Option A in Question 11 was reported in Section 4.2: the presence of a higher second moment (and all higher order even moments) in Option A of Question 9 could foster the choice of Option B in this question, while the second moments are equal between the two options in Question 11; related to the previous explanation, we can add the fact that in Question 9 there is a "safe" option (sure pay-off, higher central moments equal to zero), while it is missing in Question 11, where both options have an uncertain pay-off. Another possible explanation to this behavior could be the undervaluation of the low probability of losing money. However, if this is the case, the respondents should choose Option A in Question 9 too, underestimating also the chance in a thousand to lose $1000 \$$. Therefore, the surprising result of Question 11 should be further investigated in future experiments.

Summing up, in presence of losses (involving negative third and other odd higher moments), we observe a puzzling result of "unstable" behavior.

\section{D.3 Positive and negative odd (third) higher moments}

In this subsection, we study the joint behavior for the pairs Questions 8-9, and 10-11. Both couples contain two questions that are similar (or identical) except for the fact that one presents a gain and a positive third moment, while the other presents a loss and a negative third moment.

We start with Question 8 and 9. Table 16 reports the joint distribution of the answers to these questions. We find very clear and strong results:

- half of the sample chooses option A in Question 8 and option B in Question 9, that is they prefer the options with the higher odd central moments;

- $22 \%$ of the respondents prefer the option with the lower even moments (variance, kurtosis), that is option $\mathrm{B}$ in both questions.

- the remaining $28 \%$ that chooses option A in Question 9 can be either risk prone persons (as shown by Panel A of Table 14) or subjects who perform random choices.

Therefore, this table shows that higher odd moments are very important in the choices, and they could be even more relevant than even moments (including the second one), confirming the literature findings on "prudence" previously cited. 
Table 16: Joint distribution of replies to Questions 8 and 9.

\begin{tabular}{|l|c|c|c|}
\hline & q9-A & q9-B & Total \\
\hline q8-A & 18.83 & 49.79 & 68.62 \\
\hline q8-B & 9.41 & 21.97 & 31.38 \\
\hline Total & 28.24 & 71.76 & 100.00 \\
\hline
\end{tabular}

Table 17: Joint distribution of replies to Questions 10 and 11.

\begin{tabular}{|c|c|c|c|}
\hline & q11-A & q11-B & Total \\
\hline q10-A & 30.13 & 19.46 & 49.58 \\
\hline q10-B & 30.96 & 19.46 & 50.42 \\
\hline Total & 61.09 & 38.91 & 100.00 \\
\hline
\end{tabular}

Table 17 reports the joint distribution of the answers to Questions 10 and 11. In this case, results are less clear and far from the theoretical prediction in Scott and Horvath [1980]. Indeed, as already said, the answer to Question 11 contrasts with the theoretical prediction and the conditional distributions do not present any structure: for instance, given that a subject answers A in Question 11, the probability to choose A or B in Question 10 are equal and it holds also for people that choose Option B in Question 11. Perhaps choices are more complicated when both alternative are uncertain and present a high second moment. This feature should be further analysed in future experiments.

\section{E Results on forth moment analyses}

\section{E.1 Forth moment analysis with gains and with zero expected value}

Questions 12 and 13 study choices between two options that present different fourth (and higher) even moments. Indeed, Option A presents larger higher order even moments in both Question 12 and 13. However, Question 12 has a positive expected value (5000\$), while Question 13 presents zero expected value.

As explained in Section 4.3, for Question 12, we can observe a weak tendency to the "fourth moment risk aversion" (the so-called "temperance"). Instead in Questions 13, the majority of people (56.90\%) prefer Option A, that is the option with higher kurtosis, against the Scott and Horvath [1980] prediction. Therefore, when the expected value is not positive, people often seem to show a preference for risk (no "temperance").

Table 18 reports the joint distribution of replies to Questions 12 and 13. Even if we observe a huge degree of heterogeneity, some tendencies are observable:

- $55 \%$ of subjects are coherent in the two questions and, in particular, $29.29 \%$ choose in both cases Option A with the highest kurtosis. The coherence is confirmed observing the conditional distributions: if a subject prefers Option A in Question 13 then he/she prefers Option A also in Question 12, while if a subject prefers Option B in Question 13 then he/she prefers Option B in Question 12 too (similarly, who prefers Option A in Question 12 has a higher probability of preferring Option A also in Question 13 compared to people who prefer Option B in Question 12 , and vice versa).

- another large group (27.62\%) is composed of respondents who choose Option B in Question 
Table 18: Joint distribution of replies to Questions 12 and 13.

\begin{tabular}{|c|c|c|c|}
\hline & q13-A & q13-B & Total \\
\hline q12-A & 29.29 & 17.36 & 46.65 \\
\hline q12-B & 27.62 & 25.73 & 53.35 \\
\hline Total & 56.90 & 43.10 & 100.00 \\
\hline
\end{tabular}

12 and Option A in Question 13, that is they present "fourth moment risk aversion" ("temperance") in presence of gains, and "fourth moment risk love" when the expected value is zero.

These results are weak and similar to random choices, confirming the contrasting findings on "temperance", with values around $50 \%-50 \%$ especially if the lotteries are presented in a reduced form (Deck and Schlesinger, 2016, find 47\% of temperate choices, and ?, ?, find 50\%). A possible explanation can be given by the increasing difficulty of the questions (an increasing number of possible outcomes) when the moment increases, that induces respondents to choose a random option. However, there is a tendency towards risk aversion in presence of a positive expected value, while there is a tendency towards risk love when the expected value is zero. Given that these results have some similarities with the results on the second moment, and given that the prediction of Scott and Horvath (1980) on higher moments is based on the preference on first and second moment, in the next subsection we will study jointly the behavior in questions regarding second and fourth moments.

\section{E.2 Forth moment analysis compared with second moment analysis}

We start studying Questions 6 and 12, that is the preference of subjects for second and forth moments in presence of gains (positive expected value) with a large stake size ${ }^{13}$. The results are reported in Table 19. We find that:

- the largest group contains almost half of the sample (44.35\%) and is composed of persons that consistently choose the option with lower even moments, showing risk averse (including both "risk aversion" and "temperance") behavior;

- consistency in the preference for even moments seems to be present, as predicted by Scott and Horvath [1980]. Indeed, $57 \%$ of respondents are consistent between the two choices (the previous $44.35 \%$, plus $12.55 \%$ of persons who consistently choose the option with higher even moments, showing risk prone behavior). Moreover, the consistency between the choices on the even moments is confirmed observing the conditional distributions: if a subject prefers Option A in Question 6 then he/she tends to prefer Option A also in Question 12, while if a subject prefers Option B in Question 6 then he/she tends to prefer Option B in Question 12 too;

- there is a large group of subjects that choose Option B in Question 6 and Option A in Question 12 , that is they present a preference for lower second moment and for higher fourth moment. This feature should be further investigated in order to understand if it is a quite unexpected "real" feature or it is due to random choices;

- the second moment presents a much stronger result (very far from a 50\%-50\% random choice) probably because the impact on the choices decreases when the order of the moment increases:

${ }^{13}$ Using Question 1 instead of Question 6 produces similar results (even if slightly "weaker"). 
Table 19: Joint distribution of replies to Questions 6 and 12.

\begin{tabular}{|l|c|c|c|}
\hline & q12-A & q12-B & Total \\
\hline q6-A & 12.55 & 9.00 & 21.55 \\
\hline q6-B & 34.10 & 44.35 & 78.45 \\
\hline Total & 46.65 & 53.35 & 100.00 \\
\hline
\end{tabular}

Table 20: Joint distribution of replies to Questions 3 and 13.

\begin{tabular}{|c|c|c|c|}
\hline & q13-A & q13-B & Total \\
\hline q3-A & 23.43 & 16.11 & 39.54 \\
\hline q3-B & 33.47 & 26.99 & 60.46 \\
\hline Total & 56.90 & 43.10 & 100.00 \\
\hline
\end{tabular}

the second moment is more relevant in governing the choices than the fourth. The last feature is not surprising and can be the reason that makes many theories discard higher moments. The complexity (that can cause random choices) of the choices built in order to asses the fourth moment relevance could be a further reason for this difference.

Now, we study the joint distribution of replies to some questions regarding even moments assessment and presenting an expected value equal to zero; in particular, we will observe Questions 3, 4 and 13. We start from 3 and 13 that have large stakes. Very heterogeneous behaviors can be observed in Table 20. However, the largest group (33.47\% of subjects that choose B to Question 3 and A to Question 13) is composed of people that are risk averse in the second moment and risk prone in the fourth moment, differently from Scott and Horvath's (1980) prediction. Indeed, only half of the sample presents consistent behavior between the two even moments $(27 \%$ are consistently risk averse, and $23 \%$ are consistently risk prone).

However, Question 13 is much more similar to Question 4 (that presents a zero expected value and a low variance gap between the options) because in both questions the gap on the even moments of the two options are relatively small. As a consequence, not surprisingly, the marginal percentage obtained in the two questions are similar: 59\%-41\% for Question 4 and 57\%-43\% for Question 13. This is due to the fact that the inconsistent subjects (who choose A in a question and B in the other) are divided into two compensating groups that present almost the same weight (24.69\% choose A in Question 4 and B in Question 13, while $22.38 \%$ choose B in Question 4 and A in Question 13). Table 21 shows also that $53 \%$ of persons are consistent as theoretically predicted by Scott and Horvath (1980), but this percentage is not statistically different from a 50\%-50\% division. Moreover, 35\% of persons are consistently risk prone (they always answer A) and only $18 \%$ are consistently $\mathrm{r}$ isk averse to even moments. These results suggest two considerations: (i) lack of consistency can be related to random choices that could be driven by weak preferences, also due to small gaps between the moments; (ii) consistent risk prone behavior doubles consistent risk averse behavior hinting that with zero expected value and small gaps between the moments, people tend to be risk prone.

To summarize, subjects focus more on the second moment rather than the fourth. However, in presence of gains (positive expected values), we are able to observe a slight tendency to find consistent choices on even moments as predicted by Scott and Horvath [1980] and, in particular, people often prefer lower even moments (they are consistently risk averse). Instead, in presence of a zero expected value, we do not find the tendency to be consistent and the majority of respondents tends to be risk prone. This behavior on the 
Table 21: Joint distribution of replies to Questions 4 and 13.

\begin{tabular}{|l|c|c|c|}
\hline & q13-A & q13-B & Total \\
\hline q4-A & 34.52 & 24.69 & 59.21 \\
\hline q4-B & 22.38 & 18.41 & 40.79 \\
\hline Total & 56.90 & 43.10 & 100.00 \\
\hline
\end{tabular}

fourth moment seems very similar to the behavior on the second moment when the variance gap of the two options is low, in a sort of "extended peanuts effect". A possible explanation is that the effect of the fourth moment can be compared to the effect of low gaps in the second moment. Another possibility is that the gap between the fourth moment of the two options presented in our questions is too low, while in the presence of a larger gap subjec ts could go back to "temperance" as happened for the risk aversion on the second moment (see the discussion in Section C.3.1 regarding the results for Question 3 and 4 reported in Table 11). Further empirical studies could try to understand this feature. 